TRANSACTIONS OF THE

AMERICAN MATHEMATICAL SOCIETY

Volume 357, Number 8, Pages 3133-3167

S 0002-9947(04)03678-5

Article electronically published on December 2, 2004

\title{
VALENCE OF COMPLEX-VALUED PLANAR HARMONIC FUNCTIONS
}

\author{
GENEVRA NEUMANN
}

\begin{abstract}
The valence of a function $f$ at a point $w$ is the number of distinct, finite solutions to $f(z)=w$. Let $f$ be a complex-valued harmonic function in an open set $R \subseteq \mathbb{C}$. Let $S$ denote the critical set of $f$ and $C(f)$ the global cluster set of $f$. We show that $f(S) \cup C(f)$ partitions the complex plane into regions of constant valence. We give some conditions such that $f(S) \cup C(f)$ has empty interior. We also show that a component $R_{0} \subseteq R \backslash f^{-1}(f(S) \cup C(f))$ is an $n_{0}$-fold covering of some component $\Omega_{0} \subseteq \mathbb{C} \backslash(f(S) \cup C(f))$. If $\Omega_{0}$ is simply connected, then $f$ is univalent on $R_{0}$. We explore conditions for combining adjacent components to form a larger region of univalence. Those results which hold for $C^{1}$ functions on open sets in $\mathbb{R}^{2}$ are first stated in that form and then applied to the case of planar harmonic functions. If $f$ is a light, harmonic function in the complex plane, we apply a structure theorem of Lyzzaik to gain information about the difference in valence between components of $\mathbb{C} \backslash(f(S) \cup$ $C(f))$ sharing a common boundary arc in $f(S) \backslash C(f)$.
\end{abstract}

\section{INTRODUCTION}

We study here complex-valued harmonic functions in the plane, which we refer to simply as harmonic functions. The behavior of such a function can be vastly different from that of a holomorphic function. Analytic polynomials take every value a finite number of times. In contrast, the range of a harmonic polynomial can exclude an open region of the complex plane. Picard's theorem states that a transcendental entire function takes every value, with the exception of possibly one point, an infinite number of times. In contrast, there are transcendental harmonic functions that omit open regions. Also, there are transcendental harmonic functions that approach $\infty$ as $z \rightarrow \infty$ (like analytic polynomials), such that each $w \in \mathbb{C}$ has a finite number of distinct preimages (like analytic polynomials), and such that the maximum possible number of preimages is unbounded (like transcendental entire functions.)

A harmonic polynomial $f(z)$ is a harmonic function of the form $f(z)=p(z)+\overline{q(z)}$ where $p$ and $q$ are analytic polynomials in $z$. Let $n_{p}$ be the degree of $p$ as a polynomial in $z$ and $n_{q}$ the degree of $q$. A. Wilmshurst [Wil94 Wil98] showed that when $f$ has a finite number of zeros, it has at most $N^{2}$ distinct zeros, where $N=\max \left(n_{p}, n_{q}\right)$. Wilmshurst's bound is sharp; there are examples [BHS95 Wil98]

Received by the editors September 17, 2003.

2000 Mathematics Subject Classification. Primary 30C99, 26B99; Secondary 31A05, 26C99.

Key words and phrases. Planar harmonic functions, $C^{1}$ functions in $\mathbb{R}^{2}$, regions of constant valence.

${ }^{1}$ We will refer to Wil98] when a result appears in both [Wil94] and Wil98]. 
of harmonic polynomials with $N^{2}$ distinct zeros when $n_{p}=N$ and $n_{q}=N-1$. For $1 \leq n_{q} \leq n_{p}-1$, Wilmshurst conjectured that $f$ has at most $n_{q}\left(n_{q}-1\right)+3 n_{p}-2$ distinct zeros. D. Khavinson and G. Świątek KS03 recently proved Wilmshurst's conjecture for the case $n_{q}=1$ using methods from complex dynamics.

We will be concerned with questions related to the valence of harmonic functions. The valence of a function $f$ at a given point $w$, denoted $\operatorname{Val}(f, w)$, is the number of distinct points $z$ in the domain of $f$ such that $f(z)=w$. The valence of a function, denoted $\operatorname{Val}(f)$, is the supremum of $\operatorname{Val}(f, w)$.

Let $f(z)=(u(z), v(z))$ be a $C^{1}$ function in an open set $R \subseteq \mathbb{R}^{2}$. The Jacobian of $f$ is given by $J_{f}=u_{x} v_{y}-u_{y} v_{x}$. The inverse function theorem tells us that if $J_{f}(z) \neq 0$, then $f$ is a homeomorphism in some neighborhood of $z$. H. Lewy Lew36 proved the converse when $u$ and $v$ are both real-valued harmonic functions. Hence, if $f=u+i v$ is harmonic in an open set $R \subseteq \mathbb{C}$, then $f$ is a local homeomorphism at $z$ if and only if $J_{f}(z) \neq 0$. The critical set $S$ of a $C^{1}$ function $f$ consists of those points where the Jacobian vanishes. If $f$ is harmonic, the critical set consists of those points where $f$ is not locally $1-1$.

When studying specific examples of harmonic polynomials in $\mathbb{C}$ and looking at graphs of the image of the critical set using Mathematica ${ }^{2}$ the author noticed that the image of the critical set partitions the complex plane into regions of constant valence. This is not true for all harmonic functions. If $\lim _{z \rightarrow \infty}|f(z)| \neq \infty$, then we need to include another set in order to partition the complex plane into regions of constant valence.

Let $C(f, \infty)$ denote those finite values $w$ such that we can find a sequence $\left\{z_{n}\right\}$ with $z_{n} \rightarrow \infty$ and $f\left(z_{n}\right) \rightarrow w ; C(f, \infty)$ is the cluster set of $f$ at $\infty$. The global cluster set of a function $f$ with domain $R$ is denoted $C(f)$ and consists of all finite values which are approached on some sequence of points in $R$ which converge to a point in $\partial R \cup\{\infty\}$. (Our definitions are slightly different from those in the book of E. Collingwood and A. Lohwater [CL66], where a cluster set can include the point at infinity. We are interested in partitions of the finite complex plane and exclude the point at infinity.)

We show, under suitable conditions, that the plane can be partitioned into regions of constant valence. Since many of our results hold not only for harmonic functions but for $C^{1}$ functions in open subsets of $\mathbb{R}^{2}$, we will state the partitioning results first for $C^{1}$ functions defined in an open set in $\mathbb{R}^{2}$ and then specialize to the case where the function is harmonic in an open set in $\mathbb{C}$. We will then look at the case when $f$ is harmonic in all of $\mathbb{C}$. With each additional assumption on $f$, we can say more about the partitioning set. When $f$ is a light harmonic function, we can apply some results of A. Lyzzaik Lyz92 to show that the valences for two regions separated by an arc in the image of the critical set differ by a non-zero, even number, if that arc contains some point not in the cluster set.

Let $R_{1}$ be a component of $R \backslash\left(f^{-1}(f(S) \cup C(f))\right)$. We will see that $f\left(R_{1}\right)$ is a component of $\mathbb{R}^{2} \backslash(f(S) \cup C(f))$ and that $\left.f\right|_{R_{1}}$ is an even cover of $f\left(R_{1}\right)$ in the sense of Munkres [Mun75, p. 331]. Suppose that $R_{2}$ is another component of this partition of $R$; suppose also that $R_{2}$ shares a common boundary arc with $R_{1}$. When $f$ is harmonic, we will look at conditions for $R_{1}$ and $R_{2}$ to be mapped to different components of $\mathbb{C} \backslash(f(S) \cup C(f))$. We will also explore the behavior of $f$ on $\partial R_{1}$ (including the behavior at puncture points.) If $f$ is univalent in $R_{1}$ and in $R_{2}$, can

\footnotetext{
${ }^{2}$ Mathematica is a registered trademark of Wolfram Research, Inc.
} 
we join $R_{1}$ and $R_{2}$ along the interior of their shared boundary arc to get a larger region of univalence?

This paper is organized as follows: Section 2 contains notation and gives an overview of Lyzzaik's characterization of the local behavior of light harmonic functions. Section 3 contains partitioning results for $C^{1}$ functions in $\mathbb{R}^{2}$; it looks at a partition of $\mathbb{R}^{2}$ into regions of constant valence and at the corresponding partition of the preimage. It is also noted that many of the results can be extended to the case of $C^{1}$ functions in $\mathbb{R}^{n}$. Because some of the results in Section 3 are very basic and elementary, it is possible that they may exist somewhere in the literature. However, the author has not been able to find a reference, so detailed proofs will be presented. Section 4 applies these results to harmonic functions defined in an open set. Section 5 applies these results to the case where the harmonic function is defined in the entire complex plane. Finally, Section 6 uses our partitioning results and Lyzzaik's local characterization of light harmonic functions to relate the valences of two components in the partition of the image which share a common boundary arc.

The figures below were produced from EPS files generated by Mathematica routines 3 written by the author. Some of the figures contain alphanumeric labels; these labels were manually inserted into the EPS files.

\section{NotATion AND BACKGROUND MATERIAL}

Let $R$ be an open set in $\mathbb{R}^{2}$. Let $f: R \rightarrow \mathbb{R}^{2}$ be $C^{1}$. We can also write $f$ as $f(z)=(u(z), v(z))$, where $u, v: R \rightarrow \mathbb{R}$ and $z=(x, y) \in \mathbb{R}^{2}$. Then

$$
\begin{aligned}
J_{f}(z)= & u_{x}(z) v_{y}(z)-u_{y}(z) v_{x}(z), \\
S= & \left\{z \in R: J_{f}(z)=0\right\}, \\
C(f)= & \left\{\zeta \in \mathbb{R}^{2}: \exists\left\{z_{n}\right\}_{n=1}^{\infty} \subset R \text { with } \lim _{n \rightarrow \infty} z_{n} \in \partial R \cup\{\infty\}\right. \text { and } \\
& \left.\lim _{n \rightarrow \infty} f\left(z_{n}\right)=\zeta\right\}, \\
C(f, \infty)= & \left\{\zeta \in \mathbb{R}^{2}: \exists\left\{z_{n}\right\}_{n=1}^{\infty} \subset \mathbb{R}^{2} \text { with } \lim _{n \rightarrow \infty}\left|z_{n}\right| \rightarrow \infty\right. \text { and } \\
& \left.\lim _{n \rightarrow \infty} f\left(z_{n}\right)=\zeta\right\}, \\
B(w, \epsilon)= & \{z:|z-w|<\epsilon\}, \\
\operatorname{Val}(f, w)= & \#\{z \in R: f(z)=w\}, \\
V_{N}(f)= & \{w: w \notin f(S) \cup C(f) \text { and } \operatorname{Val}(f, w)=N\}, \\
\operatorname{Val}(f, V)= & \sup _{w \in V}\{\operatorname{Val}(f, w) .
\end{aligned}
$$

(1) $S$ denotes the critical set of $f$.

(2) $C(f)$ denotes the global cluster set in the finite plane of $\left.f\right|_{R}$. Notice that if $R=\mathbb{R}^{2}$, then $C(f)=C(f, \infty)$.

(3) $\overline{f(S)} \subseteq f(S) \cup C(f)$ : Let $\left\{w_{n}\right\}_{n=1}^{\infty} \subseteq f(S)$. Suppose that $\lim _{n \rightarrow \infty} w_{n}=w_{0}$, where $w_{0}$ is finite. For each $w_{n}$, there exists $z_{n} \in S$ such that $f\left(z_{n}\right)=$ $w_{n}$. If $\left\{z_{n}\right\}_{n=1}^{\infty}$ is unbounded, then $w_{0} \in C(f)$ by definition. If $\left\{z_{n}\right\}_{n=1}^{\infty}$ is bounded, then it has a convergent subsequence, say $\left\{z_{m}\right\}$, such that $z_{m} \rightarrow z_{0} \in \mathbb{R}^{2}$. If $z_{0} \in R$, then $z_{0} \in S$ by the continuity of $J_{f}(z)$; hence $w_{0} \in f(S)$. Otherwise, we must have that $z_{0} \in \partial R$; hence $w_{0} \in C(f)$.

(4) It is well known that $C(f)$ is closed.

(5) $f(S) \cup C(f)$ is closed, since $\overline{f(S)} \subseteq f(S) \cup C(f)$.

(6) $f^{-1}(f(S) \cup C(f))$ is relatively closed in $R$, since $f$ is continuous.

\footnotetext{
${ }^{3}$ These routines are enhanced versions of the Mathematica routines in [Neu03]. For the figures in this paper, $z \in f^{-1}(w)$ if $|\operatorname{Re} f(z)-\operatorname{Re} w|+|\operatorname{Im} f(z)-\operatorname{Im} w|<10^{-10}$.
} 
2.1. Lyzzaik's local description of light harmonic functions. Recall that a function is said to be light if the preimage of each point is empty or totally disconnected. A. Lyzzaik Lyz92] has characterized the local behavior of a function $f$ which is light and harmonic in a simply connected, open set in $\mathbb{C}$. Lyzzaik pays special attention to the behavior of $f$ on the critical set and the behavior of $f$ in a neighborhood of the critical set. Let $S$ denote the critical set of $f$.

We first note that since $f$ is harmonic in a simply connected, open set, we can find functions $h$ and $g$ holomorphic in that set such that $f(z)=h(z)+\overline{g(z)}$. Thus, $z \in S$ if and only if $0=J_{f}(z)=\left|h^{\prime}(z)\right|^{2}-\left|g^{\prime}(z)\right|^{2}$. Lyzzaik defines the meromorphic function $\psi=h^{\prime} / g^{\prime}$ and uses this function to study the local behavior of $f$ near the critical set. Note that if $z \in S$, then either $\psi(z)$ is unimodular, $\psi(z)=0$, or $\psi$ has a pole at $z$. Since $f$ is also light, $S$ has empty interior and $h^{\prime}(z)=0=g^{\prime}(z)$ at isolated points (see Lemma 4.7). Hence the zeros and poles of $\psi$ which lie in $S$ are isolated. In Lyzzaik's classification of critical points, the set of isolated critical points is denoted $N$. Since $\psi$ is unimodular at the remaining points in $S$, if $z \in S \backslash N$, then $z$ lies in an analytic arc in $S$. The set of branch points of $S$ is denoted $F_{3}$. Hence, if $z \in S \backslash\left(N \cup F_{3}\right)$, in some small neighborhood of $z$, there is a unique analytic arc in $S$ with $z$ in its interior. Lyzzaik also classifies those critical points $z \in S \backslash\left(N \cup F_{3}\right)$ where the the image of $f$ stops. Let $\gamma \subset S \backslash\left(N \cup F_{3}\right)$ be an analytic arc in $S$ such that $z \in$ int $\gamma$. Suppose that $f(\gamma)$ has zero speed at $f(z)$. If the argument of the tangent to $f(\gamma)$ jumps by $\pm \pi$ at $f(z)$, then $f$ is said to have a harmonic cusp at $f(z)$ and $z \in F_{1}$. Otherwise, if $z \in S \backslash\left(N \cup F_{1} \cup F_{3}\right)$ and $f^{\prime}(z)=0=g^{\prime}(z)$, then $z \in F_{2}$.

Lyzzaik shows that $N \cup\left(\bigcup_{j=1}^{3} F_{j}\right)$ consists of isolated points, and if $\gamma \subset S \backslash$ $\left(N \cup F_{3}\right)$, then $\left.f\right|_{\gamma}$ is a local homeomorphism. Further, if $z \in S \backslash\left(N \cup F_{1} \cup F_{3}\right)$, then a subarc of $S$ with $z$ in its interior is mapped to a convex ard 4 by $f$. Lyzzaik also shows that if $z \in F_{3}$, then $f(S)$ in some neighborhood of $z$ consists of convex arcs and harmonic cusps. Lyzzaik's classification of critical points is discussed in more detail in Section 6 below.

We will also use Lyzzaik's local characterization of $f$ in a neighborhood of $z_{0}$ for $z_{0} \in S \backslash\left(N \cup F_{3}\right)$. This result is given below as Theorem 6.6. We now review some background for this result and explain the notation.

Let $D$ denote the unit disc centered at the origin. A function $f$ is said to be locally topologically $z^{n}$ at $z_{0}$, denoted $f_{z_{0}} \sim z^{n}$, if there exist an open neighborhood $U$ of $z_{0}$ and homeomorphisms $h_{1}: U \rightarrow D$ and $h_{2}: \mathbb{C} \rightarrow \mathbb{C}$ such that $h_{2} \circ f\left(z_{0}\right)=0$ and such that $h_{2} \circ f \circ h_{1}^{-1}(\zeta)=\zeta^{n}$ for all $\zeta \in D$. A result of S. Stöilow (see Sto56] or Lemma 1 in AL88) says that if $f$ is continuous and open in a neighborhood of $z_{0}$, then $f$ is locally topologically $z^{n}$ for some positive integer $n$. Y. Abu-Muhanna and A. Lyzzaik [AL88] extend Stöilow's result to points in the boundary:

Lemma 2.1 (Abu-Muhanna and Lyzzaik). Let $G^{+}=\{z:|z|<1, \operatorname{Im} z>0\}$ be the open semi-disc and $G=\{z:|z|<1, \operatorname{Im} z \geq 0\}$ the half-closed semi-disc. Suppose that $f: G \rightarrow \mathbb{C}$ is a continuous function open in $G^{+}$and topological on $G \backslash G^{+}$. Then for every $z_{0} \in G \backslash G^{+}$there is a positive integer $n$ such that $f$ at $z_{0}$ is locally topologically $z^{2 n-1}$. This result also holds for sets homeomorphic to $G^{+}$ and $G$.

\footnotetext{
${ }^{4}$ Lyzzaik defines a convex arc to be a directed simple arc where the slope of the tangent is continuously increasing.
} 
They prove this by constructing a function $F(z)$ using an idea similar to that used in proving the Schwarz reflection principle and showing that $F$ is an open map on a disc centered at $z_{0}$. Then they apply Stöilow's result to $F$. The result follows by restricting $h_{1}$ and $F$ to $G$.

Suppose that $f$ is a harmonic function in some open set $R \subseteq \mathbb{C}$. Given $z_{0} \in R \backslash S$, $f$ is either sense-preserving $\left(J_{f}(z)>0\right)$ or sense-reversing $\left(J_{f}(z)<0\right)$ in some open neighborhood of $z_{0}$. Suppose that $f_{z_{0}} \sim z^{n}$. If we require $h_{1}$ and $h_{2}$ to be sense-preserving homeomorphisms, we see that $f_{z_{0}} \sim z^{n}$ if $f$ is sense-preserving in a punctured neighborhood of $z_{0}$ and that $f_{z_{0}} \sim \bar{z}^{n}$ if $f$ is sense-reversing in a punctured neighborhood of $z_{0}$.

We now suppose that $f$ is a light harmonic function in some simply connected, open set. Suppose that $z_{0} \in$ int $\gamma_{0} \subset S \backslash\left(F_{3} \cup N\right)$. Then $z_{0}$ can be thought of as living in a shared boundary arc of two adjacent regions. In a sufficiently small neighborhood $U$ of $z_{0}, f$ is 1-1 on $\gamma=\gamma_{0} \cap U$ (recall that $\left.f\right|_{\gamma}$ is a local homeomorphism at $z_{0}$ ) and $\gamma$ splits $U$ into a sense-preserving region $R^{+}$and a sense-reversing region $R^{-}$. Lyzzaik [Lyz92] notes that Lemma 2.1] applies to $R^{+} \cup \gamma$ and to $R^{-} \cup \gamma$. The notation $f_{z_{0}} \sim z^{j}, \bar{z}^{k}$ means that $f$ at $z_{0}$ is locally topologically $z^{j}$ in $R^{+} \cup \gamma$ and that $f$ at $z_{0}$ is locally topologically $\bar{z}^{k}$ in $R^{-} \cup \gamma$. Lemma 2.1 also shows that $j$ and $k$ must both be odd positive integers. A structure theorem of Lyzzaik for this case (Theorem 6.6 below) gives the values for $j$ and $k$ based on various conditions on $z_{0}$.

In particular, suppose that $z_{0} \in S \backslash\left(N \cup\left(\bigcup_{i=1}^{3} F_{i}\right)\right)$. Then Theorem 6.6 gives $f_{z_{0}} \sim z, \bar{z}$ since $g^{\prime}\left(z_{0}\right) \neq 0$. We can assign a direction to $f(\gamma)$, which is part of the common boundary of $f\left(R^{+}\right)$and $f\left(R^{-}\right)$. We see that the image of $U$ is folded over $f(\gamma)$; in other words, $f(U \backslash \gamma)$ lies to one side of $f(\gamma)$. The tangent line to $f(\gamma)$ at $f\left(z_{0}\right)$ lies in $f(U)$. One way to see this is to recall that $f$ is univalent and sense-preserving in $R^{+}\left(f_{z_{0}} \sim z\right.$ for $z \in R^{+}$.) Also, $f$ is locally 1-1 on $\gamma$. A result of P. Duren and D. Khavinson [DK97] shows that $f(\gamma)$ is concave with respect to $f\left(R^{+}\right)$; hence the tangent line to $f(\gamma)$ at $f\left(z_{0}\right)$ lies in $f(U)$.

In Remark 5.19, we note that Lyzzaik's result implies that two components of $\mathbb{C} \backslash f^{-1}(f(S) \cup C(f, \infty))$ which share a common boundary arc in $S$ must be mapped to the same component of $\mathbb{C} \backslash(f(S) \cup C(f, \infty))$. In Section 6, we use Lyzzaik's structure theorem to compare the valence in two components of $\mathbb{C} \backslash(f(S) \cup C(f, \infty))$ when the two components share a common boundary arc in $f(S)$.

\section{Partitioning Results For $C^{1}$ MAPPings in $\mathbb{R}^{2}$}

We show that $f(S) \cup C(f)$ partitions $\mathbb{R}^{2}$ into regions of constant valence when $f: R \rightarrow \mathbb{R}^{2}$ is $C^{1}$ in an open set $R \subseteq \mathbb{R}^{2}$. We then examine a corresponding partition of $R$.

\subsection{Regions of constant valence for $C^{1}$ mappings in $\mathbb{R}^{2}$.}

Lemma 3.1. Let $R$ be open in $\mathbb{R}^{2}$. Let $f: R \rightarrow \mathbb{R}^{2}$ be $C^{1}$. Let $w_{0} \in \mathbb{R}^{2} \backslash \overline{f(S)}$. Suppose that $\operatorname{Val}\left(f, w_{0}\right) \geq N_{0} \geq 0$, where $N_{0}$ is finite. Then there exists an open neighborhood of $w_{0}$, say $W_{0}$, such that $\operatorname{Val}(f, w) \geq N_{0}$ for all $w \in W_{0}$.

Proof. Trivial if $\overline{f(S)}=\mathbb{R}^{2}$, so suppose that $\mathbb{R}^{2} \backslash \overline{f(S)} \neq \varnothing$. The result is also trivial if $N_{0}=0$, so we will suppose that $N_{0}>0$. Since $\operatorname{Val}\left(f, w_{0}\right) \geq N_{0}$, choose $N_{0}$ distinct points $z_{1}, \ldots, z_{N_{0}}$ in $f^{-1}\left(w_{0}\right)$. By assumption, these points will be in the open set $R \backslash f^{-1}(\overline{f(S)})$. By the inverse function theorem, we may find an open 
neighborhood of each $z_{j}$, say $U_{j}$, such that $f: U_{j} \rightarrow W_{j}$ is 1-1 and onto, where $W_{j}$ is an open neighborhood of $w_{0}$. Since $N_{0}$ is finite, we can choose the $U_{j}$ to be pairwise disjoint. Let $W_{0}=\bigcap_{j=1}^{N_{0}} W_{j}$. Then $W_{0}$ is an open, non-empty set such that each $w \in W_{0}$ has a distinct preimage in each $U_{j}$. The lemma then follows.

Lemma 3.2. Let $R$ be an open set in $\mathbb{R}^{2}$. Let $f: R \rightarrow \mathbb{R}^{2}$ be $C^{1}$. Fix $N_{0} \geq 0$, finite. Then $V_{N_{0}}(f)$ is open in $\mathbb{R}^{2}$.

Proof. Let $Q_{0}=\mathbb{R}^{2} \backslash(f(S) \cup C(f))$. Then $Q_{0}$ is open. By definition, $V_{N_{0}}(f) \subseteq Q_{0}$. If $V_{N_{0}}(f)=\varnothing$, the claim is vacuously true. Suppose that $V_{N_{0}}(f) \neq \varnothing$; hence we also have $Q_{0} \neq \varnothing$. Choose $w_{0} \in V_{N_{0}}(f)$. Thus, $\operatorname{Val}\left(f, w_{0}\right)=N_{0}$ and $w_{0} \in Q_{0}$.

We first note that there exists an open neighborhood $\tilde{B} \subseteq Q_{0}$ of $w_{0}$ such that $\operatorname{Val}(f, w) \geq N_{0}$ for all points $w \in \tilde{B}$. Since $\overline{f(S)} \subseteq f(S) \cup C(f)$, this follows from Lemma 3.1

Since $\tilde{B}$ is open, we may choose $\epsilon_{0}>0$ such that $B\left(w_{0}, \epsilon_{0}\right) \subseteq \tilde{B}$. Suppose that we can find $\left\{\epsilon_{j}\right\}_{j=1}^{\infty}$ with $\lim _{j \rightarrow \infty} \epsilon_{j}=0$, such that for each $j, 0<\epsilon_{j}<\epsilon_{0}$ and there exists $w_{j} \in B\left(w_{0}, \epsilon_{j}\right)$ with $\operatorname{Val}\left(f, w_{j}\right)>N_{0}$. By construction, $w_{j} \rightarrow w_{0}$ and $\operatorname{Val}\left(f, w_{j}\right) \geq N_{1}$ where $N_{1}=N_{0}+1$. Without loss of generality, we may suppose that the $w_{j}$ are distinct. We will show that $\operatorname{Val}\left(f, w_{0}\right) \geq N_{1}>N_{0}=\operatorname{Val}\left(f, w_{0}\right)$, a contradiction.

We may find $z_{j 1}, z_{j 2}, \ldots, z_{j N_{1}} \in R \backslash f^{-1}(f(S) \cup C(f))$, distinct, such that $f\left(z_{j k}\right)$ $=w_{j}$ for $k=1,2, \ldots, N_{1}$, since $w_{j} \notin f(S) \cup C(f)$ and $\operatorname{Val}\left(f, w_{j}\right) \geq N_{1}$. Moreover, $\bigcup_{k=1}^{N_{1}}\left\{z_{j k}\right\}_{j=1}^{\infty}$ consists of pairwise distinct points, since the $w_{j}$ are distinct.

(1) $\bigcup_{k=1}^{N_{1}}\left\{z_{j k}\right\}_{j=1}^{\infty}$ is bounded: This is obvious if $R$ is bounded. So, suppose that $R$ is unbounded. Suppose that $\bigcup_{k=1}^{N_{1}}\left\{z_{j k}\right\}_{j=1}^{\infty}$ is unbounded. Thus, we may find $\left\{z_{j_{l} k_{l}}\right\}_{l=1}^{\infty}$ where $k_{l} \in\left\{1,2, \ldots, N_{1}\right\}$ such that $\left|z_{j_{l} k_{l}}\right| \rightarrow \infty$ as $l \rightarrow \infty$. By the pigeonhole principle, we may find a subsequence where $k_{l}$ is fixed; i.e., $\left\{z_{l N}\right\}_{l=1}^{\infty}$ for some $N \in\left\{1,2, \ldots, N_{1}\right\}$ such that $\left|z_{l N}\right| \rightarrow \infty$ as $l \rightarrow \infty$. Since $\left\{z_{l N}\right\}_{l=1}^{\infty} \subseteq\left\{z_{j N}\right\}_{j=1}^{\infty}$ and $\lim _{j \rightarrow \infty} f\left(z_{j N}\right)=\lim _{j \rightarrow \infty} w_{j}=$ $w_{0}, \lim _{l \rightarrow \infty} f\left(z_{l N}\right)=w_{0}$. Since $\left|z_{l N}\right| \rightarrow \infty, w_{0} \in C(f)$. But $C(f) \cap Q_{0}=\varnothing$ and $w_{0} \in Q_{0}$ by assumption. Thus $\bigcup_{k=1}^{N_{1}}\left\{z_{j k}\right\}_{j=1}^{\infty}$ is bounded.

(2) If $z$ is a finite cluster point of $\bigcup_{k=1}^{N_{1}}\left\{z_{j k}\right\}_{j=1}^{\infty}$, then $z \in R$ and $f(z)=w_{0}$ : Find $\left\{z_{j_{l} k_{l}}\right\}_{l=1}^{\infty}$ where $k_{l} \in\left\{1,2, \ldots, N_{1}\right\}$ such that $\lim _{l \rightarrow \infty} z_{j_{l} k_{l}}=z$. As above, we may find a subsequence of this sequence with $k_{l}$ constant, say $\left\{z_{l N}\right\}_{l=1}^{\infty}$ such that $z_{l N} \rightarrow z$. Since $\left\{z_{l N}\right\}_{l=1}^{\infty} \subseteq\left\{z_{j N}\right\}_{j=1}^{\infty}$ and $f\left(z_{j N}\right) \rightarrow w_{0}$, $\lim _{l \rightarrow \infty} f\left(z_{l N}\right)=w_{0}$. Since $z$ is a cluster point of a subset of $R$, either $z \in R$ or $z \in \partial R$. If $z \in \partial R$, we have $w_{0} \in C(f)$, a contradiction. Hence $z \in R$. Since $z_{l N} \rightarrow z$ and $f$ is continuous at $z, f(z)=w_{0}$.

(3) $\bigcup_{k=1}^{N_{1}}\left\{z_{j k}\right\}_{j=1}^{\infty}$ has at least $N_{1}$ distinct cluster points in $R$ : By (1), all of the cluster points are finite. By (2), all of these cluster points are in $R$. Let $z$ be a cluster point. Suppose that for each $\epsilon>0$, there is a $j>0$ such that $\left|z_{j k_{1}}-z\right|<\epsilon$ and $\left|z_{j k_{2}}-z\right|<\epsilon$ for some choice of $k_{1}, k_{2}$ with $k_{1} \neq k_{2}$. But for each such $j, f\left(z_{j k_{1}}\right)=w_{j}=f\left(z_{j k_{2}}\right)$, with $z_{j k_{1}} \neq z_{j k_{2}}$, so $f$ is not locally $1-1$ at $z$; hence $z \in S$. By (2), $f(z)=w_{0} \in Q_{0}$ with $Q_{0} \cap f(S)=\varnothing$, so $z \notin S$, a contradiction. So, there exists $\epsilon>0$ where for each $j>0$ such that $\left|z_{j k}-z\right|<\epsilon$ holds, it holds for exactly one value of $k$ for that choice of $j$. Thus we must have at least $N_{1}$ cluster points in $R$. 
From (1)-(3), we see that $w_{0}$ has at least $N_{1}$ distinct preimages in $R$, which gives the desired contradiction. With this contradiction, we have shown that $\exists \epsilon>0$ such that $\operatorname{Val}(f, w)=N_{0}$ for all $w \in B\left(w_{0}, \epsilon\right)$. Since $w_{0} \in V_{N_{0}}(f)$ is arbitrary, $V_{N_{0}}(f)$ is open in $\mathbb{R}^{2}$.

Lemma 3.3. Let $f$ be a $C^{1}$ mapping defined in an open set $R \subseteq \mathbb{R}^{2}$. If $\operatorname{Val}\left(f, w_{0}\right)=$ $\infty$, then $w_{0} \in f(S) \cup C(f)$.

Proof. Since $\operatorname{Val}\left(f, w_{0}\right)=\infty$, we may choose a sequence $\left\{z_{n}\right\} \subseteq f^{-1}\left(w_{0}\right)$ consisting of distinct points. If $\left\{z_{n}\right\} \subseteq f^{-1}\left(w_{0}\right)$ has a bounded subsequence (which we also denote by $\left.\left\{z_{n}\right\}\right)$ converging to a point $z^{*} \in R$, we will show that $z^{*} \in S$. Given $\epsilon>0$, we can find $N$ such that $\left|z_{n}-z^{*}\right|<\epsilon$ for all $n>N$. By continuity, $f\left(z^{*}\right)=w_{0}$. Hence $f$ is not locally $1-1$ at $z^{*}$. By the inverse function theorem, $J_{f}\left(z^{*}\right)=0$. Hence $z^{*} \in S$ and $w_{0}=f\left(z^{*}\right) \in f(S)$.

Otherwise, we may suppose that either $\left\{z_{n}\right\}$ has a subsequence converging to a finite point in $\partial R$ or has an unbounded subsequence. In either case, since each point of the subsequence is mapped to $w_{0}$, we have $w_{0} \in C(f)$.

Theorem 3.4. Let $R$ be open in $\mathbb{R}^{2}$. Let $f: R \rightarrow \mathbb{R}^{2}$ be $C^{1}$. Then $f(S) \cup C(f)$ partitions $\mathbb{R}^{2}$ into regions of constant valence.

Proof. Let $\varphi(w)=\operatorname{Val}(f, w)$. It is enough to show that $\varphi$ is a continuous, integervalued function at each $w \in \mathbb{R}^{2} \backslash(f(S) \cup C(f))$. Choose $w_{0} \in \mathbb{R}^{2} \backslash(f(S) \cup C(f))$. By Lemma 3.3. $\varphi\left(w_{0}\right)$ is a finite integer, say $j$. Hence $w_{0} \in V_{j}(f)$. By Lemma 3.2. $V_{j}(f)$ is open. By definition, $V_{j} \subseteq \mathbb{R}^{2} \backslash(f(S) \cup C(f))$. Hence there exists $\delta>0$ such that $B\left(w_{0}, \delta\right) \subseteq V_{j}$ and $\varphi(w)=j$ for all $w \in B\left(w_{0}, \delta\right)$. Thus $\varphi$ is a continuous, integervalued function in every region off $f(S) \cup C(f)$ and the conclusion follows.

From Lemma 3.1 and Theorem 3.4, we see that $\operatorname{Val}(f, w)$ is lower semi-continuous on $\mathbb{R}^{2} \backslash \overline{f(S)}$ for points with finite valence.

Example 3.5. $f(x, y)=\left(x^{2}+y^{2}, 2 x y\right)$.

Here $R=\mathbb{R}^{2}$, so $C(f)=C(f, \infty)$. Clearly $|f| \rightarrow \infty$ as $z \rightarrow \infty$, so $C(f, \infty)=\varnothing$. The critical set consists of the lines $y=x$ and $y=-x . f$ maps the critical set to the rays $y=x$ and $y=-x$ for $x \geq 0$. A calculation shows that $f(S)$ partitions $\mathbb{R}^{2}$ into regions of constant valence. In particular, each point with $x>0$ and $|y|<x$ has four distinct preimages. The origin has one preimage. Each point in the image of the critical set in the right half plane has two preimages. The remaining points have no preimages. Note that the behavior of $\operatorname{Val}(f, w)$ on the partitioning set is consistent with $\operatorname{Val}(f, w)$ being lower semi-continuous on $\mathbb{R}^{2} \backslash \overline{f(S)}$. If we rewrite $f$ as $f=u+i v$ where $z=x+i y$, it is clear that $f(z)$ is not harmonic.

We can say a little about the behavior of $\operatorname{Val}(f, w)$ when $\operatorname{Val}(f, w)$ is infinite.

Lemma 3.6. Let $R$ be open in $\mathbb{R}^{2}$. Let $f: R \rightarrow \mathbb{R}^{2}$ be $C^{1}$. Suppose that $\operatorname{Val}\left(f, w_{0}\right)=\infty$ and that $f^{-1}\left(w_{0}\right) \backslash S$ contains an infinite number of distinct points. Then, given $N_{0} \geq 0$, finite, there exists an open neighborhood of $w_{0}$, say $W_{0}$, such that $\operatorname{Val}(f, w) \geq N_{0}$ for all $w \in W_{0}$.

Proof. Obvious if $N_{0}=0$, so assume $N_{0}>0$. Choose $\left\{z_{1}, \ldots, z_{N_{0}}\right\} \subseteq f^{-1}\left(w_{0}\right) \backslash S$, distinct. By the inverse function theorem, for each $z_{j}$, there exists an open neighborhood of $z_{j}$, say $B_{j}$, such that $f$ is $1-1$ on $B_{j}$ and $f\left(B_{j}\right)$ is open. We may choose the $B_{j}$ so that they are pairwise disjoint. Let $W_{0}=\bigcap f\left(B_{j}\right)$. 
Example 4.4 below demonstrates why we must require that $w_{0}$ have an infinite number of distinct preimages off of the critical set. In this example, the origin has infinite valence and every neighborhood of the origin contains a point with no preimages. However, the preimages of the origin all lie in the critical set.

Theorem 3.7. Let $f$ be a $C^{1}$ mapping in an open set $R \subseteq \mathbb{R}^{2}$. Let $V$ be a connected component of $\mathbb{R}^{2} \backslash(f(S) \cup C(f))$ and let $N_{0}=\operatorname{Val}(f, V)$. Let $w_{0} \in \partial V \backslash \overline{f(S)}$. Then $\operatorname{Val}\left(f, w_{0}\right) \leq N_{0}$.

Proof. By Theorem 3.4 and Lemma 3.3, $f$ has constant, finite valence on $V$; hence $N_{0}$ is finite. Suppose that $\operatorname{Val}\left(f, w_{0}\right)>N_{0}$. Then, by Lemma 3.1, there exists an open neighborhood $V_{0}$ of $w_{0}$ such that $\operatorname{Val}(f, w)>N_{0}$ for all $w \in V_{0}$. Since $w_{0} \in \partial V, V_{0} \cap V$ is a non-empty open set. For $w \in V_{0} \cap V \subseteq V, \operatorname{Val}(f, w)>N_{0}=$ $\operatorname{Val}(f, w)$, a contradiction and the theorem follows.

The preceding two results show that $\operatorname{Val}(f, w)$ is lower semi-continuous off of $\overline{f(S)}$. Consider $f(z)=z+R e e^{z}$ in Example 4.2 below. Since $R=\mathbb{C}$, $C(f)=C(f, \infty)$. Each $w \in f(S) \cup C(f, \infty)$ has exactly one preimage. Each point in $f(S)$ has a neighborhood containing points with no preimages and points with two preimages, so this example shows why $f(S)$ is excluded in the preceding result. If $w_{0} \in C(f, \infty), w_{0}$ lies in a horizontal line separating a region where $\operatorname{Val}(f, w)=2$ from a region where $\operatorname{Val}(f, w)=1$. Thus, for all $w$ in a sufficiently small neighborhood of $w_{0}, \operatorname{Val}(f, w) \geq \operatorname{Val}\left(f, w_{0}\right)=1$, in accord with the result above.

Remark 3.8. The results above are vacuous if the partitioning set $f(S) \cup C(f)$ fills the plane. When does the partitioning set have empty interior? If $\operatorname{int}(f(S))=\varnothing$, $f(S) \cup C(f)$ will have empty interior iff $\operatorname{int}(C(f))=\varnothing$. Why? Let $U$ be an open subset of $f(S) \cup C(f)$. Since $C(f)$ is closed, $U \backslash C(f)$ is an open subset of $f(S)$. Since $f(S)$ has empty interior, either $U=\varnothing$ or $U$ is a non-empty subset of $C(f)$.

Theorem 3.9. Let $R$ be open in $\mathbb{R}^{2}$. Let $f: R \rightarrow \mathbb{R}^{2}$ be a $C^{1}$ mapping such that $S$ is nowhere dense. Suppose that $C(f)$ has non-empty interior. Then points with infinite valence are dense in the interior of $C(f)$.

Proof. Suppose not. Then we can choose $w_{0} \in \operatorname{int} C(f)$ and some $\epsilon_{0}>0$ such that $f$ has finite valence at each point in $B\left(w_{0}, \epsilon_{0}\right)$.

Since $w_{0}$ is a cluster point of $f$, there exist $\left\{z_{n}\right\} \subset R$ and $z_{0} \in \partial R \cup\{\infty\}$ such that $z_{n} \rightarrow z_{0}$ and $f\left(z_{n}\right) \rightarrow w_{0}$. Since $w_{0}$ has finite valence, we may choose $z_{N}$ such that $w_{0} \neq f\left(z_{N}\right) \in B\left(w_{0}, \frac{\epsilon_{0}}{2}\right) \subset B\left(w_{0}, \epsilon_{0}\right)$. If $z_{N} \notin S$, let $\zeta_{1}=z_{N}$. Otherwise, by the continuity of $f$ in the open set $R, \exists \delta>0$ such that $B\left(z_{N}, \delta\right) \subset R$ and $f\left(B\left(z_{N}, \delta\right)\right) \subset B\left(w_{0}, \frac{\epsilon_{0}}{2}\right)$. Since $f$ has finite valence at each point in $B\left(w_{0}, \epsilon_{0}\right)$ and since $S$ is nowhere dense in $R$, choose $\zeta_{1} \in B\left(z_{N}, \delta\right) \backslash S$ such that $f\left(\zeta_{1}\right) \neq w_{0}$. Let $w_{1}=f\left(\zeta_{1}\right)$. Since $f$ is continuous at $\zeta_{1}$, we may choose $0<\epsilon_{1}<\frac{\epsilon_{0}}{2}$ and $0<\delta_{1}<\operatorname{dist}\left(\zeta_{1}, \partial R\right) / 2$ such that $f\left(B\left(\zeta_{1}, \delta_{1}\right)\right) \subset \overline{B\left(w_{1}, \epsilon_{1}\right)} \subset B\left(w_{0}, \epsilon_{0}\right)$. Since $\zeta_{1} \in R \backslash S$, by the inverse function theorem, we can find non-empty open subsets $U_{1} \subseteq B\left(\zeta_{1}, \delta_{1}\right)$ and $V_{1} \subseteq B\left(w_{1}, \epsilon_{1}\right)$ such that $\zeta_{1} \in U_{1}, w_{1} \in V_{1}$ and $f: U_{1} \rightarrow V_{1}$ is 1-1, onto. Moreover, $f\left(U_{1}\right)=V_{1} \subset B\left(w_{0}, \epsilon_{0}\right) \subset C(f)$.

Repeat the preceding argument with $w_{1}$ in place of $w_{0}$ to find $z_{N} \in R \backslash B\left(\zeta_{1}, \delta_{1}\right)$ such that $f\left(z_{N}\right) \notin\left\{w_{0}, w_{1}\right\}$ and $f\left(z_{N}\right) \in V_{1}$. This gives us $\zeta_{2} \in B\left(z_{N}, \delta\right) \backslash S$ such 
that $w_{2}=f\left(\zeta_{2}\right) \notin\left\{w_{0}, w_{1}\right\}$. Arguing as above, we may choose $0<\epsilon_{2}<\epsilon_{1}$ such that $\overline{B\left(w_{2}, \epsilon_{2}\right)} \subset V_{1}$. Similarly, we may choose $0<\delta_{2}<\operatorname{dist}\left(\zeta_{2}, \partial R\right) / 2$ such that $B\left(\zeta_{2}, \delta_{2}\right) \cap B\left(\zeta_{1}, \delta_{1}\right)=\varnothing$ and such that $f\left(B\left(\zeta_{2}, \delta_{2}\right)\right) \subseteq B\left(w_{2}, \epsilon_{2}\right)$. As above, we can find non-empty open subsets $U_{2} \subseteq B\left(\zeta_{2}, \delta_{2}\right) \subset R$ and $V_{2} \subseteq B\left(w_{2}, \epsilon_{2}\right)$ such that $\zeta_{2} \in U_{2}, w_{2} \in V_{2}$, and $f: U_{2} \rightarrow V_{2}$ is $1-1$, onto. Moreover, $f\left(U_{2}\right)=V_{2} \subseteq$ $B\left(w_{2}, \epsilon_{2}\right) \subset V_{1} \subset B\left(w_{1}, \epsilon_{1}\right) \subset B\left(w_{0}, \epsilon_{0}\right) \subset C(f)$. By construction, $U_{1} \cap U_{2}=\varnothing$.

Continue in this manner to get a sequence of nested non-empty open sets $V_{n} \subseteq$ $B\left(w_{n}, \epsilon_{n}\right)$ with $\overline{B\left(w_{n}, \epsilon_{n}\right)} \subset B\left(w_{n-1}, \epsilon_{n-1}\right)$ such that $w_{n} \rightarrow w^{*}=\bigcap \overline{B\left(w_{n}, \epsilon_{n}\right)} \subset$ $B\left(w_{0}, \epsilon_{0}\right)$. Thus, $\operatorname{Val}\left(f, w^{*}\right)$ is finite. But $w^{*}$ has a preimage in each $U_{n}$ where the $U_{n}$ are by construction pairwise disjoint. This contradicts $w^{*}$ having a finite number of distinct preimages in $R$.

We are not claiming that points with infinite valence are only in the interior of $C(f)$. In Example 4.4 the origin has infinite valence and is in $C(f)$. However, $C(f)$ has empty interior.

3.2. Partitioning the preimage by $f^{-1}(f(S) \cup C(f))$. Suppose that $R$ is an open set in $\mathbb{R}^{2}$ and that $f: R \rightarrow \mathbb{R}^{2}$ is $C^{1}$. Recall that $f(S) \cup C(f)$ is closed. We have seen that $f(S) \cup C(f)$ partitions the plane into components of constant valence. What does this tell us about the behavior of $f$ in a component of $R \backslash f^{-1}(f(S) \cup C(f))$ ?

Theorem 3.10. Let $R$ be an open set in $\mathbb{R}^{2}$. Suppose that $f: R \rightarrow \mathbb{R}^{2}$ is a $C^{1}$ mapping. Let $R_{0}$ be a connected component of $R \backslash f^{-1}(f(S) \cup C(f))$ and choose $z_{0} \in$ $R_{0}$. Let $w_{0}=f\left(z_{0}\right)$ and choose the connected component $\Omega_{0} \subseteq \mathbb{R}^{2} \backslash(f(S) \cup C(f))$ such that $w_{0} \in \Omega_{0}$. Then $f\left(R_{0}\right)=\Omega_{0}$.

Proof. Note that the result holds vacuously if $f^{-1}(f(S) \cup C(f))=R$. Suppose that $R \backslash f^{-1}(f(S) \cup C(f)) \neq \varnothing$.

We first show that $f\left(R_{0}\right) \subseteq \Omega_{0}$. Since $R_{0}$ is connected and $f$ is continuous, $f\left(R_{0}\right)$ is connected. Since $w_{0} \in f\left(R_{0}\right)$, by our choice of $\Omega_{0}, f\left(R_{0}\right) \subseteq \Omega_{0}$.

It remains to show that $\Omega_{0} \backslash f\left(R_{0}\right)$ is empty. Suppose not. Since $R_{0}$ is open and $R_{0} \cap S$ is empty, $f$ is an open map on $R_{0}$; hence $f\left(R_{0}\right)$ is open. Since $f\left(R_{0}\right)$ is open and a proper subset of the component $\Omega_{0}, \exists \tilde{w} \in \Omega_{0} \cap\left(\overline{f\left(R_{0}\right)} \backslash f\left(R_{0}\right)\right)$. By Lemma 3.3, $\operatorname{Val}\left(f, w_{0}\right)$ is finite. Suppose that $\operatorname{Val}\left(f, w_{0}\right)=N_{0}$. By Theorem 3.4 the valence of $f$ is constant in $\Omega_{0}$; hence $\operatorname{Val}(f, \tilde{w})=N_{0}$. Thus $\exists z_{1}, \ldots, z_{N_{0}}$ distinct in $R \backslash f^{-1}(f(S) \cup C(f))$ such that $f\left(z_{j}\right)=\tilde{w}$ for $j=1, \ldots, N_{0}$. Since $\tilde{w} \notin f\left(R_{0}\right)$, $\left\{z_{1}, \ldots, z_{N_{0}}\right\} \cap R_{0}=\varnothing$.

For each $z_{j}$, we may find an open neighborhood of $z_{j}$ in $R$, say $B_{j}$, such that $B_{j} \cap S=\varnothing, B_{j} \cap R_{0}=\varnothing$, and such that $f$ is 1-1 and an open map on $B_{j}$. We may choose the $B_{j}$ to be pairwise disjoint. Then $V=\bigcap_{j=1}^{N_{0}} f\left(B_{j}\right)$ is open and non-empty. Also, $\tilde{B}_{j}=f^{-1}(V) \cap B_{j}$ is an open neighborhood of $z_{j}$ where $f$ is 1-1 and such that $\tilde{B}_{j} \cap R_{0}$ is empty. Since $\tilde{w} \in V$ and $\tilde{w} \in \overline{f\left(R_{0}\right)}, \exists w \in f\left(R_{0}\right)$ such that $w \in V$. Thus, each of the pairwise disjoint $\tilde{B}_{j}$ contains one preimage of $w$. But, $R_{0}$ also contains at least one preimage of $w$. Thus, $\operatorname{Val}(f, w) \geq N_{0}+1$. However, $w \in \Omega_{0}$, so $\operatorname{Val}(f, w)=N_{0}$, a contradiction. Thus, $f\left(R_{0}\right)=\Omega_{0}$.

Lemma 3.11. Let $f, R_{0}, \Omega_{0}$, and $w_{0}$ be as in Theorem 3.10, Suppose that $w_{0}$ has exactly $n_{0}$ distinct preimages in $R_{0}$, where $0<n_{0} \leq \operatorname{Val}\left(f, w_{0}\right)$. Then every $w \in \Omega_{0}$ has exactly $n_{0}$ distinct preimages in $R_{0}$. 
Proof. This proof was suggested by D. Sarason. By Theorem 3.4 and Lemma 3.3, $\operatorname{Val}(f, w)=\operatorname{Val}\left(f, w_{0}\right)=N_{0}<\infty$ for all $w \in \Omega_{0}$. By Theorem 3.10, $1 \leq$ $\operatorname{Val}\left(\left.f\right|_{R_{0}}, w\right) \leq N_{0}$ for all $w \in \Omega_{0}$. Let

$$
W_{j}=\left\{w \in \Omega_{0}: \operatorname{Val}\left(\left.f\right|_{R_{0}}, w\right)=j\right\} .
$$

Then $\Omega_{0}=\bigcup_{j=1}^{N_{0}} W_{j}$. Clearly, the $W_{j}$ are pairwise disjoint. If the $W_{j}$ are open, then only one of the $W_{j}$ is non-empty since $\Omega_{0}$ is connected. Further, since $\operatorname{Val}\left(\left.f\right|_{R_{0}}, w_{0}\right)=n_{0}$, the result follows.

It remains to show that $W_{j}$ is open for $j>0$. This follows from Lemma 3.2 if $W_{j}=V_{j}\left(\left.f\right|_{R_{0}}\right)$. By construction, the critical set of $\left.f\right|_{R_{0}}$ is empty; hence $V_{j}\left(\left.f\right|_{R_{0}}\right)=$ $\left\{w \notin C\left(\left.f\right|_{R_{0}}\right): \operatorname{Val}\left(\left.f\right|_{R_{0}}, w\right)=j\right\}$. We need to show that $C\left(\left.f\right|_{R_{0}}\right)$ is disjoint from $\Omega_{0}$. Let $\left\{z_{n}\right\} \subset R_{0}$ converge to a point $z_{0} \in \partial R_{0} \cup\{\infty\}$. If $z_{0} \in \partial R \cup\{\infty\}$, then if $\left\{\left.f\right|_{R_{0}}\left(z_{n}\right)\right\}$ has a finite cluster point, this point is in $C(f)$, hence not in $\Omega_{0}$. On the other hand, if $z_{0} \in R$, then $f$ is continuous at $z_{0} \in f^{-1}(f(S) \cup C(f))$ and $\left\{\left.f\right|_{R_{0}}\left(z_{n}\right)\right\}$ converges to a point in $f(S) \cup C(f)$, which, again, is not in $\Omega_{0}$. Thus $V_{j}\left(\left.f\right|_{R_{0}}\right)=W_{j}$ and $W_{j}$ is open.

Thus, $f^{-1}(f(S) \cup C(f))$ partitions $R$ into components, each of which is mapped onto a component of $\mathbb{R}^{2} \backslash(f(S) \cup C(f))$ by $f$. We will now show that $f$ is a covering map on each component of this partition of the preimage. First, we recall a standard result (see Mun75], page 341):

Theorem 3.12 (Munkres). Let $p:\left(E, e_{0}\right) \rightarrow\left(B, b_{0}\right)$ be a covering map. If $E$ is path connected, then there is a surjection $\phi: \pi_{1}\left(B, b_{0}\right) \rightarrow p^{-1}\left(b_{0}\right)$. If $E$ is simply connected, $\phi$ is a bijection.

Theorem 3.13. Let $R$ be an open set in $\mathbb{R}^{2}$. Suppose that $f: R \rightarrow \mathbb{R}^{2}$ is $C^{1}$. Let $R_{0}$ be a connected component of $R \backslash f^{-1}(f(S) \cup C(f))$. Choose $z_{0} \in R_{0}$ and let $w_{0}=f\left(z_{0}\right)$. Suppose that $w_{0}$ has exactly $n_{0}$ distinct preimages in $R_{0}$. Then $R_{0}$ is a $n_{0}$-fold covering of $\Omega_{0}$. Moveover, if $\Omega_{0}$ is simply connected, then $f$ is univalent in $R_{0}$.

Proof. We first show that $f: R_{0} \rightarrow \Omega_{0}$ is a covering map. By Theorem $3.10 f$ is onto; $f$ is continuous by assumption. Choose $w_{0} \in \Omega_{0}$. By Lemma 3.11, $w_{0}$ has $n_{0}$ distinct preimages in $R_{0}$. Constructing $V$ and the $\tilde{B}_{j}$ as in the proof of Theorem 3.10 (except that we choose $B_{j} \subset R_{0}$ ), each of the pairwise disjoint open sets $\tilde{B}_{j}$ is homeomorphic to $V$ since $R_{0} \cap S=\varnothing$. Also, by Lemma 3.11, each $w \in V$ has exactly $n_{0}$ distinct preimages in $R_{0}$. Thus, $R_{0} \cap f^{-1}(V)=\bigcup_{j=1}^{n_{0}} \tilde{B}_{j}$. Thus, $V$ is evenly covered by $\left.f\right|_{R_{0}}$. Since $w_{0}$ is arbitrary, $\left.f\right|_{R_{0}}$ is a covering map and $R_{0}$ is a covering space of $\Omega_{0}$. Since $f^{-1}(w)$ has $n_{0}$ distinct elements in $R_{0}$ for each $w \in \Omega_{0}$, $R_{0}$ is a $n_{0}$-fold covering of $\Omega_{0}$.

We note that $R \backslash f^{-1}(f(S) \cup C(f))$ is open in $\mathbb{R}^{2}$. Hence the component $R_{0}$ is open in $\mathbb{R}^{2}$. Since $R_{0}$ is an open, connected subset of $\mathbb{R}^{2}$, given any two points in $R_{0}$, we may find a polygonal path contained in $R_{0}$ that joins the two points. Hence, $R_{0}$ is path connected. Choose $w_{0} \in \Omega_{0}$. By Theorem 3.12, there is a surjection $\phi: \pi_{1}\left(\Omega_{0}, w_{0}\right) \rightarrow f^{-1}\left(w_{0}\right)$, where we are restricting $f$ to $R_{0}$. If we also assume that $\Omega_{0}$ is simply connected, then $\pi_{1}\left(\Omega_{0}, w_{0}\right)$ is the trivial group. Since $\phi$ is a surjection, $w_{0}$ must have exactly one preimage in $R_{0}$. Hence $n_{0}=1$ and $f$ is univalent on $R_{0}$. 
Example 3.14. $f(x, y)=(x \cos y, y)$.

Here, $R=\mathbb{R}^{2}$ and $C(f)=C(f, \infty)$. If we rewrite $f$ as $f=u+i v$ where $z=x+i y$, it is clear that $f(z)$ is $C^{1}$ but not harmonic. A calculation shows that

$$
\begin{aligned}
& S=\left\{\left(x, \frac{(2 k+1) \pi}{2}\right): x \in \mathbb{R} \text { and } k \in \mathbb{Z}\right\}, \\
& f(S)=\left\{\left(0, \frac{(2 k+1) \pi}{2}\right): k \in \mathbb{Z}\right\} .
\end{aligned}
$$

Also, $C(f, \infty)$ consists of the horizontal lines $y=\frac{(2 k+1) \pi}{2}$ where $k$ is an integer. Each $w \in \mathbb{R}^{2} \backslash(f(S) \cup C(f, \infty))$ has exactly one preimage. Each $w \in f(S)$ has an infinite number of preimages. Each $w \in C(f, \infty) \backslash f(S)$ has no preimages. Note that if we fix $a \in \mathbb{R}$ and let $y_{n}=\cos ^{-1}\left(a / x_{n}\right)$, then $f\left(x_{n}, y_{n}\right) \rightarrow\left(a, \frac{(2 k+1) \pi}{2}\right) \in C(f, \infty)$ as $x_{n} \rightarrow \infty$, provided that we choose the branch of $\cos ^{-1}$ such that $\cos ^{-1}\left(a / x_{n}\right) \rightarrow$ $\frac{(2 k+1) \pi}{2}$.

The partitioning set of our domain is $f^{-1}(f(S) \cup C(f, \infty)$ ), which is $S$ (a collection of horizontal lines). It is clear that if $(x, y) \notin S$, then $f(x, y) \neq\left(0, \frac{2 k+1}{2} \pi\right)$. If we choose $w=(a, b) \in \mathbb{R}^{2}$ such that $w \notin f(S) \cup C(f, \infty)$, then $b \neq \frac{2 k+1}{2} \pi$. It is clear that $w$ has exactly one preimage; namely, $(a / \cos b, b)$ and that this preimage point does not lie in $S$. Hence, $f^{-1}(f(S) \cup C(f, \infty))$ partitions $\mathbb{R}^{2}$ into horizontal strips where $f$ is univalent.

3.3. Adjacent components of the preimage. A non-empty, connected set is said to be degenerate if it consists of a single point. If two distinct components of our partition of $R$ share a non-degenerate common boundary arc in $R$, will $f$ map both to the same component of $\mathbb{R}^{2} \backslash(f(S) \cup C(f))$ ?

Lemma 3.15. Let $R \subseteq \mathbb{R}^{2}$ be open. Let $f: R \rightarrow \mathbb{R}^{2}$ be $C^{1}$. Let $R_{1}$ and $R_{2}$ be distinct components of $R \backslash f^{-1}(f(S) \cup C(f))$ such that $\overline{R_{1}} \cap \overline{R_{2}} \neq \varnothing$. Suppose that $f\left(R_{1}\right)=f\left(R_{2}\right)=\Omega$. If $\partial \Omega \cap$ int $\bar{\Omega}=\varnothing$, then there exists no non-empty set $\gamma \subseteq\left(\overline{R_{1}} \cap \overline{R_{2}} \cap R\right) \backslash S$ such that $R_{1} \cup R_{2} \cup \gamma$ is open.

Proof. By contradiction. Suppose that $R_{1}$ and $R_{2}$ are disjoint components of $R \backslash f^{-1}(f(S) \cup C(f))$ such that $f\left(R_{1}\right)=f\left(R_{2}\right)$. Suppose also that there exists $\gamma \subseteq\left(\overline{R_{1}} \cap \overline{R_{2}} \cap R\right) \backslash S$ such that $\gamma \neq \varnothing$ and such that $R_{1} \cup R_{2} \cup \gamma$ is open. By Theorem 3.10 there exist $\Omega_{1}, \Omega_{2}$, components of $\mathbb{R}^{2} \backslash(f(S) \cup C(f))$, such that $f\left(R_{1}\right)=\Omega_{1}$ and $f\left(R_{2}\right)=\Omega_{2}$. By assumption, $\Omega_{1}=\Omega_{2}=\Omega$.

Since $R_{1}$ and $R_{2}$ are disjoint components of $R \backslash f^{-1}(f(S) \cup C(f))$ and $\gamma \subseteq$ $\overline{R_{1}} \cap \overline{R_{2}} \cap R, \gamma \subseteq f^{-1}(f(S) \cup C(f))$. Thus $f(\gamma) \subseteq f(S) \cup C(f)$. By continuity, $f(\gamma) \subseteq \bar{\Omega}$, so $f(\gamma) \subseteq \partial \Omega$. Now let $R_{0}=R_{1} \cup R_{2} \cup \gamma$. By assumption, $R_{0}$ is open. Since $R_{0} \cap S=\varnothing, f$ is an open map on $R_{0}$ by the inverse function theorem and $f\left(R_{0}\right)$ is open. But $f\left(R_{0}\right)=f\left(R_{1}\right) \cup f\left(R_{2}\right) \cup f(\gamma)=\Omega \cup f(\gamma)$ and we have seen that $f(\gamma) \subseteq \partial \Omega$. Since $f\left(R_{0}\right)$ is open, $f\left(R_{0}\right) \subseteq$ int $\bar{\Omega}$. Choose $z_{0} \in \gamma$ (recall that $\left.\gamma \neq \varnothing\right)$. Then $f\left(z_{0}\right) \in \partial \Omega \cap f\left(R_{0}\right) \subseteq \partial \Omega \cap$ int $\bar{\Omega}$. Thus $\partial \Omega \cap$ int $\bar{\Omega} \neq \varnothing$, a contradiction.

Lemma 3.16. Let $R \subseteq \mathbb{R}^{2}$ be open. Let $f: R \rightarrow \mathbb{R}^{2}$ be a light $C^{1}$ function. Let $R_{1}$ and $R_{2}$ be distinct components of $R \backslash f^{-1}(f(S) \cup C(f))$ such that $\overline{R_{1}} \cap \overline{R_{2}} \neq \varnothing$. Suppose that $f\left(R_{1}\right)=f\left(R_{2}\right)=\Omega$. If $\partial \Omega \cap i n t \bar{\Omega}$ consists of a finite number of points, then there exists no non-empty, non-degenerate connected set $\gamma \subseteq\left(\overline{R_{1}} \cap \overline{R_{2}} \cap R\right) \backslash S$ such that $R_{1} \cup R_{2} \cup \gamma$ is open. 
Proof. By contradiction. Suppose that $R_{1}$ and $R_{2}$ are disjoint components of $R \backslash f^{-1}(f(S) \cup C(f))$ such that $f\left(R_{1}\right)=f\left(R_{2}\right)$. Suppose also that there exists $\gamma \subseteq\left(\overline{R_{1}} \cap \overline{R_{2}} \cap R\right) \backslash S$ such that $\gamma \neq \varnothing$ and such that $R_{0}=R_{1} \cup R_{2} \cup \gamma$ is open.

Let $P=\partial \Omega \cap$ int $\bar{\Omega}$. By assumption, $P=\left\{w_{1}, w_{2}, \ldots, w_{n}\right\}$. If $n=0$, the result follows from Lemma 3.15. Assume that $n>0$. By assumption, $\gamma$ is a non-empty, non-degenerate connected set. Since $f$ is continuous, $f(\gamma)$ is connected. So, if $f(\gamma) \subseteq P$, then $f(\gamma)=\left\{w_{j}\right\}$ for some fixed value of $j$. This contradicts $f$ being a light mapping. Thus $f(\gamma) \backslash P \neq \varnothing$. Choose $w \in f(\gamma) \backslash P$. By the arguments used in the proof of Lemma [3.15, $f(\gamma) \subseteq \partial \Omega$. As in Lemma 3.15 $f\left(R_{0}\right)$ is open and $w \in$ int $\bar{\Omega}$. Thus $w \in \partial \Omega \cap$ int $\bar{\Omega}=P$, a contradiction.

Corollary 3.17. Let $f$ be a light $C^{1}$ function in $\mathbb{R}^{2}$. Let $\Omega$ be a component of $\mathbb{R}^{2} \backslash(f(S) \cup C(f, \infty))$. Suppose that $R_{1}$ and $R_{2}$ are two distinct components of $\mathbb{R}^{2} \backslash f^{-1}(f(S) \cup C(f, \infty))$ such that $f\left(R_{1}\right)=f\left(R_{2}\right)=\Omega$.

(1) If $\partial \Omega \cap i n t \bar{\Omega}$ consists of a finite number of points, then $\left(\overline{R_{1}} \cap \overline{R_{2}}\right) \backslash S$ contains no non-degenerate connected set $\gamma$ such that $R_{1} \cup R_{2} \cup \gamma$ is open.

(2) Suppose that $\partial \Omega \cap i n t \bar{\Omega}$ consists of a finite number of points. Also suppose that (int $\left.\overline{R_{j}}\right) \cap \partial \overline{R_{j}}$ consists of a finite number of points and that $\partial\left(\right.$ int $\left.\overline{R_{j}}\right)$ is a Jordan curve for $j=1,2$. If $\overline{R_{1}} \cap \overline{R_{2}}$ contains a non-degenerate Jordan arc, then this arc is in $S$.

Proof. Using the notation of the preceding two lemmas, $R=\mathbb{R}^{2}$. So $\overline{R_{1}} \cap \overline{R_{2}} \cap R=$ $\overline{R_{1}} \cap \overline{R_{2}}$. The first claim follows from Lemma 3.16 .

The second claim follows by noting that each region $R_{j}$ is a Jordan region that has a finite number of puncture points. We are assuming that the boundaries intersect in a non-degenerate Jordan $\operatorname{arc} \rho$. Since we can find subregions of the two regions, each having $\rho$ as part of its boundary, that are Jordan regions (no punctures), $R_{1} \cup R_{2} \cup$ int $\rho$ is open. By the first claim, we must have that (int $\left.\rho\right) \backslash S$ is totally disconnected. Since $S$ is closed, $\rho \subseteq S$.

3.4. Behavior in shared boundaries and "puncture points". Let $f$ be a $C^{1}$ function on some open set $R \subseteq \mathbb{R}^{2}$. Suppose that we have a collection of components of $R \backslash f^{-1}(f(S) \cup C(f))$ where $f$ is univalent on each component. Can we combine these components to get a larger region where $f$ is univalent? The results in the preceding section (such as Corollary 3.17) handle components that share a common boundary arc in $R$, but say nothing about the behavior on the boundary arc.

If $R_{0}$ is one such component, we want to know more about the behavior of $f$ at points in $\partial R_{0} \cap R$. These could be points in boundary arcs shared with other components or could be "puncture points." A "puncture point" is an isolated point in $\partial R_{0}$. Does $f$ map $\partial R_{0}$ onto $\partial\left(f\left(R_{0}\right)\right)$ ? What can we say about $\operatorname{Val}\left(\left.f\right|_{\partial R_{0}}, w\right)$ for $w \in \partial\left(f\left(R_{0}\right)\right)$ ?

Lemma 3.18. Let $R \subseteq \mathbb{R}^{2}$ be open. Let $f$ be $C^{1}$ in $R$. Let $R_{0} \subset \mathbb{R}^{2}$ be a component of $R \backslash f^{-1}(f(S) \cup C(f))$. Then $f\left(R \cap \partial R_{0}\right) \subseteq f(R) \cap \partial\left(f\left(R_{0}\right)\right)$.

Proof. By Theorem [3.10, there exists a component of $\mathbb{R}^{2} \backslash(f(S) \cup C(f))$, say $\Omega_{0}$, such that $f\left(R_{0}\right)=\Omega_{0}$. Thus $\partial\left(f\left(R_{0}\right)\right)=\partial \Omega_{0}$. Requiring that $R_{0}$ is a proper subset of $\mathbb{R}^{2}$ guarantees that $\partial R_{0} \neq \varnothing$ in the finite plane. If $\partial R_{0} \subseteq \partial R$, the result holds vacuously. Suppose that $R \cap \partial R_{0} \neq \varnothing$. 
Since $R \cap \partial R_{0} \subseteq f^{-1}(f(S) \cup C(f)), f\left(R \cap \partial R_{0}\right) \subseteq f(S) \cup C(f)$. Fix $z_{0} \in$ $R \cap \partial R_{0}$. Then there exists a sequence $\left\{z_{n}\right\} \subset R_{0}$ such that $z_{n} \rightarrow z_{0}$. Since $z_{0} \in R$, by the continuity of $f$ at $z_{0}, f\left(z_{n}\right) \rightarrow f\left(z_{0}\right)$. But $f\left(z_{n}\right) \in \Omega_{0}$ for all $n$ and $f\left(z_{0}\right) \in f(S) \cup C(f)$. Hence $f\left(z_{0}\right) \in f(R) \cap \overline{\Omega_{0}} \cap(f(S) \cup C(f)) \subseteq f(R) \cap \partial \Omega_{0}$, so $f\left(R \cap \partial R_{0}\right) \subseteq f(R) \cap \partial \Omega_{0}$.

Lemma 3.19. Let $f$ be $C^{1}$ in $\mathbb{R}^{2}$. Let $R_{0}$ be a bounded component of $\mathbb{R}^{2} \backslash f^{-1}(f(S)$ $\cup C(f, \infty))$. Then $f\left(\partial R_{0}\right)=\partial\left(f\left(R_{0}\right)\right)$.

Proof. By Theorem [3.10 there exists a component of $\mathbb{R}^{2} \backslash(f(S) \cup C(f, \infty))$, say $\Omega_{0}$, such that $f\left(R_{0}\right)=\Omega_{0}$. Thus $\partial\left(f\left(R_{0}\right)\right)=\partial \Omega_{0}$. By Lemma 3.18, we have $f\left(\partial R_{0}\right) \subseteq f\left(\mathbb{R}^{2}\right) \cap \partial \Omega_{0}$. So $f\left(\partial R_{0}\right) \subseteq \partial \Omega_{0}$.

We now show that $\partial \Omega_{0} \subseteq f\left(\partial R_{0}\right)$. Fix $w_{0} \in \partial \Omega_{0}$. Then there exists a sequence $\left\{w_{n}\right\} \subset \Omega_{0}$ such that $w_{n} \rightarrow w_{0}$. Since $f\left(R_{0}\right)=\Omega_{0}$, there exist $z_{n} \in R_{0}$ such that $f\left(z_{n}\right)=w_{n}$ for each $n$. By assumption $R_{0}$ is bounded, so $\overline{R_{0}}$ is compact and $\left\{z_{n}\right\}$ has a convergent subsequence $\left\{z_{n_{k}}\right\}$. Since $w_{n} \rightarrow w_{0}, w_{n_{k}} \rightarrow w_{0}$. Let $z_{n_{k}} \rightarrow$ $z_{0} \in \overline{R_{0}}$. Since $f$ is continuous in $\mathbb{R}^{2}, f\left(z_{0}\right)=w_{0}$. Since $w_{0} \in f(S) \cup C(f, \infty)$, $z_{0} \in f^{-1}(f(S) \cup C(f, \infty))$. Thus $z_{0} \in \overline{R_{0}} \cap\left(f^{-1}(f(S) \cup C(f, \infty))\right)=\partial R_{0}$. Since $w_{0} \in \partial \Omega_{0}$ is arbitrary, we have $\partial \Omega_{0} \subseteq f\left(\partial R_{0}\right)$. The result follows.

Notation 3.20. Let $\Omega \subseteq \mathbb{R}^{2}$ and $f$ be a function defined on $R \subseteq \mathbb{R}^{2}$. Recall that $\operatorname{Val}\left(\left.f\right|_{R}, \Omega\right)$ denotes the valence of $\left.f\right|_{R}$ in $\Omega$. Note that if the valence of $\left.f\right|_{R}$ is constant and finite in $\Omega$, then $\operatorname{Val}\left(\left.f\right|_{R}, \Omega\right)=\operatorname{Val}\left(\left.f\right|_{R}, w\right)$ for any choice of $w \in \Omega$.

Theorem 3.21. Let $f$ be $C^{1}$ in $\mathbb{R}^{2}$. Let $R$ be a component of $\mathbb{R}^{2} \backslash f^{-1}(f(S) \cup$ $C(f, \infty))$ and let $\Omega$ be a component of $\mathbb{R}^{2} \backslash(f(S) \cup C(f, \infty))$ such that $f(z) \in \Omega$ for some $z \in R$. Choose $w \in \partial \Omega$ such that (i) $w \notin C\left(\left.f\right|_{R}, \infty\right)$, (ii) $f^{-1}(w) \cap \partial R \cap S=$ $\varnothing$, (iii) $f^{-1}(w) \cap \partial R \neq \varnothing$, and such that (iv) for each $\zeta \in f^{-1}(w) \cap \partial R$, there exists a neighborhood of $\zeta$, say $U_{\zeta}$, such that $f\left(U_{\zeta} \backslash R\right) \cap \Omega=\varnothing$. Then $w$ has exactly $\operatorname{Val}\left(\left.f\right|_{R}, \Omega\right)$ distinct preimages on $\partial R$.

Proof. By Theorem 3.10, $f(R)=\Omega$. Moreover, by Lemma 3.11, if $\eta \in \Omega$ and if $N=\operatorname{Val}\left(\left.f\right|_{R}, \eta\right)$, then $\operatorname{Val}\left(\left.f\right|_{R}, \Omega\right)=N$. Choose $w \in \partial \Omega$ satisfying the given conditions. By condition (iii), $w$ has at least one preimage on $\partial R$. Note that if $R$ is bounded, this condition follows from Lemma 3.19, Suppose that $w$ has $M$ distinct preimages on $\partial R$.

We first show that $M \geq N$ : Since $w \in \partial \Omega$, we may choose $\left\{w_{j}\right\} \subset \Omega$, where the $w_{j}$ are distinct, such that $w_{j} \rightarrow w$. For each $j$, there exist $z_{j 1}, z_{j 2}, \ldots, z_{j N}$ distinct in $R$ such that $f\left(z_{j k}\right)=w_{j}$. Let $Q=\bigcup_{k=1}^{N}\left\{z_{j k}\right\}_{j=1}^{\infty}$. So $Q \subset R$. Using arguments analogous to those used to prove Lemma 3.2, we see that $Q$ has at least $N$ distinct cluster points. We also see that the cluster points of $Q$ are in $\partial R$. Hence $M \geq N$.

We now show that $M \leq N$ : Suppose not. Then we can find distinct values $z_{1}, \ldots, z_{N+1}$ such that $z_{j} \in \partial R$ and $f\left(z_{j}\right)=w$. By (ii), $z_{j} \notin S$. So, for each $j$, we may find some neighborhood of $z_{j}$, say $V_{j}$, where $f$ is a homeomorphism. We may choose the $V_{j}$ so that they are pairwise disjoint. By (iv), for each $z_{j}$, there exists a neighborhood $U_{j}$ of $z_{j}$ such that the preimages in $U_{j}$ of all points in $f\left(U_{j}\right) \cap \Omega$ lie in $R$. Let $B_{j}=U_{j} \cap V_{j}$. Then the $B_{j}$ are pairwise disjoint and each $B_{j}$ is a non-empty, open neighborhood of $z_{j}$ where $f$ is a homeomorphism. Since $z_{j} \in \partial R$, $B_{j} \cap R \neq \varnothing$; hence $f\left(B_{j}\right) \cap \Omega \neq \varnothing$. By construction, all preimages in $B_{j}$ of points in $f\left(B_{j}\right) \cap \Omega$ lie in $R$. Since $z_{j} \in B_{j}$ and $f\left(z_{j}\right)=w, \bigcap_{j=0}^{N+1} f\left(B_{j}\right) \neq \varnothing$. 
Choose $\eta \in \Omega \cap\left(\bigcap_{j=1}^{N+1} f\left(B_{j}\right)\right)$. Then $\eta$ has a distinct preimage in each $B_{j}$, giving a total of $N+1$ distinct preimages in $\bigcup_{j=1}^{N+1} B_{j}$. Further, each of these preimages lies in $R$. But, $\eta$ has exactly $N$ distinct preimages in $R$, a contradiction. Thus, $M=N$ and $w$ has exactly $\operatorname{Val}\left(\left.f\right|_{R}, \Omega\right)$ distinct preimages on $\partial R$.

\section{Remark 3.22. We note that}

(1) We will apply this result to prove Lemma 5.15 below.

(2) The condition $w \notin C\left(\left.f\right|_{R}, \infty\right)$ automatically holds if either $C(f, \infty)$ is empty or if $R$ is bounded.

Theorem 3.23. Let $f$ be a light, $C^{1}$ function in $\mathbb{R}^{2}$. Let $R_{1}$ and $R_{2}$ be disjoint bounded components of $\mathbb{R}^{2} \backslash f^{-1}(f(S) \cup C(f, \infty))$. Suppose that $R_{1}$ and $R_{2}$ each have at most a finite number of puncture points. Suppose that for each component $\Omega \subseteq \mathbb{R}^{2} \backslash(f(S) \cup C(f, \infty)), \partial \Omega \cap i n t \bar{\Omega}$ consists of a finite number of points. Suppose that $f$ is univalent on $R_{1}$ and on $R_{2}$. Let $\gamma$ be a non-degenerate, simple arc in $\partial R_{1} \cap \partial R_{2}$. Suppose that $f($ int $\gamma)$ is a simple arc. If $S \cap$ int $\gamma=\varnothing$, then

(1) $f\left(R_{1}\right)$ and $f\left(R_{2}\right)$ are distinct components of $\mathbb{R}^{2} \backslash(f(S) \cup C(f, \infty))$.

(2) $f$ is a univalent on $R_{1} \cup R_{2} \cup$ int $\gamma$.

Proof. By construction, the combined region, $R_{1} \cup R_{2} \cup$ int $\gamma$, is open (see the proof of Corollary 3.17). The first claim follows from Lemma 3.16, To prove the second claim, we need to show that $f$ is univalent on int $\gamma$. Then $f$ is univalent in the combined region (since no point in $R_{1} \cup R_{2}$ is mapped to $f($ int $\gamma$ )). Note that $f($ int $\gamma)$ is non-degenerate since $f$ is light.

We now check that $f$ is univalent on int $\gamma$. Suppose not. Then we have two distinct points $\zeta_{1}, \zeta_{2} \in$ int $\gamma$ such that $f\left(\zeta_{1}\right)=f\left(\zeta_{2}\right)=w_{0} \in f(S) \cup C(f, \infty)$. Since $S \cap$ int $\gamma=\varnothing$, we can find a neighborhood $B_{1}$ of $\zeta_{1}$ and a neighborhood $B_{2}$ of $\zeta_{2}$ where $f$ is an open map. We may assume that these neighborhoods are disjoint and are contained in the combined region. However, $f\left(B_{1}\right) \cap f\left(B_{2}\right)$ is an open neighborhood of $w_{0}$. So it contains a point $w \in \Omega_{1}$. By construction, $w$ has at least two distinct preimages in $R_{1}$ (one in $B_{1} \cap R_{1}$ and another in $B_{2} \cap R_{1}$ ), which contradicts the univalence of $f$ in $R_{1}$.

3.5. Extension of partitioning results to $C^{1}$ mappings in $\mathbb{R}^{n}$. The notation defined at the beginning of Section 2 for the critical set and cluster set has obvious generalizations to the case of $C^{1}$ functions defined in an open set in $\mathbb{R}^{n}$, where $n$ is a positive integer. Moreover, the comments at the beginning of Section 2 concerning the properties of these sets hold. For the most part, the proofs in Section 3 do not make use of special properties of $\mathbb{R}^{2}$. Thus, the partitioning results above can be extended to $C^{1}$ functions defined in open subsets of $\mathbb{R}^{n}$, with the exceptions of Lemma 3.16, Corollary 3.17 and Theorem 3.23.

Lemma 3.16 and the first part of Corollary 3.17 hold in $\mathbb{R}^{n}$ for $n>1$. The hypothesis concerning the non-degenerate set $\gamma$ cannot hold for $n=1$. The proofs of Theorem 3.23 and of the second part of Corollary 3.17 use Jordan curves and Jordan regions; these are $n=2$ results.

\section{Partitioning Results for harmonic MAPpings in AN OPEN SET}

We will apply our results about $C^{1}$ functions in an open subset of the real plane to the case where our function is harmonic in an open subset of the complex 
plane. Unless explicitly stated otherwise, a harmonic function is a complex-valued harmonic function.

Theorem 4.1. Let $f$ be a harmonic mapping in an open set $R \subseteq \mathbb{C}$. Then $f(S) \cup$ $C(f)$ partitions $\mathbb{C}$ into regions of constant valence.

Proof. Identify $\mathbb{R}^{2}$ with $\mathbb{C}$ by putting $x=\operatorname{Re} z$ and $y=\operatorname{Im} z$. Let $u=\operatorname{Re} f$, $v=\operatorname{Im} f$. Then apply Theorem 3.4 .

Analogs to the other results in the preceding section are shown similarly.

Example 4.2. A transcendental harmonic function with finite valence:

$$
f(z)=z+\operatorname{Re} e^{z}
$$

This example was given by D. Bshouty, W. Hengartner and M. Naghibi-Beidokhti in [BHN99] as an example of a 2-valent, transcendental harmonic mapping in $\mathbb{C}$. Calculations show that

$$
C(f, \infty)=\left\{\zeta: \operatorname{Im} \zeta=\frac{(2 k+1) \pi}{2}, k \in \mathbb{Z}\right\}
$$

and

$$
S=\left\{z: \operatorname{Re} e^{z}=-1\right\} .
$$

Letting $z=x+i y$ and fixing $\zeta=a+i b, f(z)=\zeta$ when $y=b$ and $x+e^{x} \cos b=a$. The behavior of $\varphi(x)=x+e^{x} \cos b$ depends on the sign of $\cos b$ and determines the number of preimages of $\zeta$. One can then check that $f(S) \cup C(f, \infty)$ partitions $\mathbb{C}$ into regions of constant valence. In particular, each $w \in f(S) \cup C(f, \infty)$ has exactly one preimage. If $\operatorname{Im}(w) \bmod 2 \pi \in(-\pi / 2, \pi / 2), w$ also has exactly one preimage. If $\operatorname{Im}(w) \bmod 2 \pi \in(\pi / 2,3 \pi / 2)$, then $w$ has two distinct preimages if $w$ lies to the left of $f(S)$ and no preimages if $w$ lies to the right of $f(S)$. Note that if $\zeta=$ $a+i \frac{(2 k+1) \pi}{2} \in C(f, \infty)$, then $f\left(z_{n}\right) \rightarrow \zeta$ for $z_{n}=x_{n}+i \cos ^{-1}\left(\left(a-x_{n}\right) \exp \left(-x_{n}\right)\right)$ with $x_{n} \rightarrow \infty$ and the proper choice for the branch of $\cos ^{-1}$.

Example 4.3. A finite valence harmonic polynomial with an unbounded critical set:

$$
f(z)=2\left(\operatorname{Re}\left(z^{3}\right)+i z\right) .
$$

One can explicity check that

$$
\{\zeta: \operatorname{Im} \zeta=0\} \cup\left\{\zeta: \operatorname{Re} \zeta=\frac{(\operatorname{Im} \zeta)^{3}}{4}+\frac{1}{3 \operatorname{Im} \zeta}\right\}
$$

partitions $\mathbb{C}$ into regions of constant valence.

One can also check that $S=\left\{z: x y=-\frac{1}{6}\right.$, where $x=\operatorname{Re} z$ and $\left.y=\operatorname{Im} z\right\}$ and that $C(f, \infty)=\{\zeta: \operatorname{Im} \zeta=0\}$. For example, fix $a \in \mathbb{R}$; then $f\left(z_{n}\right) \rightarrow a \in C(f, \infty)$ if $z_{n}=x_{n}+i\left\{a / 2-\left(1 /\left(3 x_{n}\right)\right)\right\}$ and $x_{n} \rightarrow 0$.

A calculation shows that $f(S)=\left\{2(\operatorname{Re}(z))^{3}+\frac{1}{6 \operatorname{Re}(z)}+2 i \operatorname{Re}(z): \operatorname{Re}(z) \neq 0\right\}$, which is equivalent to $\left\{\zeta: \operatorname{Re} \zeta=\frac{(\operatorname{Im} \zeta)^{3}}{4}+\frac{1}{3 \operatorname{Im} \zeta}\right\}$. Thus $f(S) \cup C(f, \infty)$ partitions $\mathbb{C}$ into regions of constant valence. Each $w \in C(f, \infty)$ has exactly one preimage, as does each $w \in f(S)$. Looking at $f(S)$ in $\mathbb{R}^{2}$, one sees that it consists of two parabola-like curves pointing sideways. Each $w$ in the region "inside" these curves has no preimage. Outside of this region, each $w \notin(f(S) \cup C(f, \infty))$ has two distinct preimages.

Since $C(f, \infty) \neq \varnothing, f$ is an example of a finite valence harmonic polynomial where $f$ does not go to $\infty$ as $z \rightarrow \infty$. 
It is easy to check that $f^{-1}(f(S))=S$. Another easy calculation shows that $f^{-1}(C(f, \infty))$ is the imaginary axis. Recall that $w \notin f(S) \cup C(f, \infty)$ has either zero or exactly two distinct preimages. Suppose we choose $w \notin f(S) \cup C(f, \infty)$ such that $\operatorname{Val}(f, w)=2$. Note that $\operatorname{Im} w \neq 0$, since $w \notin C(f, \infty)$. Since $f(x, y)=2\left(x^{3}-3 x y^{2}-y+i x\right), \operatorname{Re}\left(f^{-1}(w)\right)=\frac{1}{2} \operatorname{Im} w$. Since $w \notin f(S)$, $\operatorname{Im}\left(f^{-1}(w)\right) \neq-1 /(3 \operatorname{Im} w)$. The vertical line $\operatorname{Re} z=\frac{1}{2} \operatorname{Im} w$ intersects $S$ at $\left(\frac{1}{2} \operatorname{Im} w,-1 /(3 \operatorname{Im} w)\right)$. The two preimages of $w$ are the endpoints of a vertical line segment bisected by $S$. From this, we see that $f^{-1}(f(S) \cup C(f, \infty))$ partitions $\mathbb{C}$ into regions where $f$ is univalent.

Example 4.4. $f(z)=2\left[\operatorname{Re}\left(z^{2}\right)+i(\operatorname{Re}(z)-\operatorname{Im}(z))\right]$.

One can readily check that $S=\{z: \operatorname{Re}(z)=\operatorname{Im}(z)\}$ and $f(S)=\{0\}$. Further, $C(f, \infty)$ is the real axis. If we rewrite $f$ letting $z=x+i y$, we see that $f(x, y)=$ $2(x-y)(x+y+i)$. A calculation shows that if $w \notin \mathbb{R}$, then $w$ has exactly one preimage. If $w$ is a non-zero real, it has no preimage. And, the origin is a point of infinite valence. Also, if we fix $a \in \mathbb{R}$ and let $y_{n}=x_{n}-\left(a /\left(4 x_{n}\right)\right)$, then $f\left(z_{n}\right) \rightarrow a \in C(f, \infty)$ for $z_{n}=x_{n}+i y_{n}$ as $\left|x_{n}\right| \rightarrow \infty$.

Example 4.5. $f(z)=z^{2}+z-\bar{z}$.

An easy calculation shows that $S=\left\{z:\left|z+\frac{1}{2}\right|=\frac{1}{2}\right\}$. This circle is mapped to the hypocycloid in Figure 1. Clearly, $C(f, \infty)=\varnothing$.

D. Sarason (personal communication) has analyzed the behavior of $f$. Among many observations about $f$, he shows that $f$ is $1-1$ on $S, 4$-valent in the bounded component of $\mathbb{C} \backslash f(S)$, and 2-valent on the portion of the real axis in the unbounded component. Now consider our partition of the domain (by $f^{-1}(f(S)$ ), since $C(f, \infty)=\varnothing)$; this is shown in Figure 2. Sarason's analysis shows that $f$ is a 1-1 mapping of the interior of each of the bounded components onto the bounded component of $\mathbb{C} \backslash f(S)$; hence $f$ is univalent on each of the bounded components of the partition of the domain. He also showed that all $g(z)=p(z)-\bar{z}$, where $\operatorname{deg} p=2$, can be reduced to $f(z)$ by means of affine transformations of the domain and range.

By Theorem 4.1, $f$ must be 2-valent on the unbounded component of $\mathbb{C} \backslash f(S)$ since real values in the unbounded component of $\mathbb{C} \backslash f(S)$ are 2-valent. It follows from Lemma 3.11 that $f$ must be $2: 1$ on the unbounded component of $\mathbb{C} \backslash f^{-1}(f(S)$ ).

It is nicest when our partitioning set $f(S) \cup C(f)$ has empty interior. Note that the results above are vacuous if the partitioning set fills the complex plane. For example, $f(z)=e^{z}$ is harmonic in $\mathbb{C}$. It is easy to see that $C(f)=\mathbb{C}$. In this case, $\operatorname{Val}(f, w)=\infty$ if $w \neq 0$ and $\operatorname{Val}(f, 0)=0$.

Remark 4.6. If $z_{0} \in R \subseteq \mathbb{C}$, where $R$ is open, then there exists a simply connected, open set $U \subseteq R$ such that $z_{0} \in U$ (for example, $B\left(z_{0}, \delta\right)$ for $\delta>0$ sufficiently small). Suppose that $f$ is harmonic in $R$. Since $U$ is simply connected, there exist functions $h$ and $g$ holomorphic in $U$ such that $f=h+\bar{g}$ in $U$. We have $J_{f}=\left|h^{\prime}\right|^{2}-\left|g^{\prime}\right|^{2}$ in $U$. Thus, $z \in S \cap U$ iff $\left|h^{\prime}(z)\right|=\left|g^{\prime}(z)\right|$. If $R$ is a simply connected, open set, then we can take $U=R$.

Lemma 4.7. Let $f$ be harmonic in a simply connected, open set $R \subseteq \mathbb{C}$. If int $S \neq$ $\varnothing$, then $f(z)=\alpha+\beta u(z)$, where $u$ is a real harmonic function and $\alpha, \beta$ are complex constants. Moreover, $S=R$ and $\operatorname{Val}\left(f, w_{0}\right)=\infty$ for every $w_{0} \in f(R)$. 


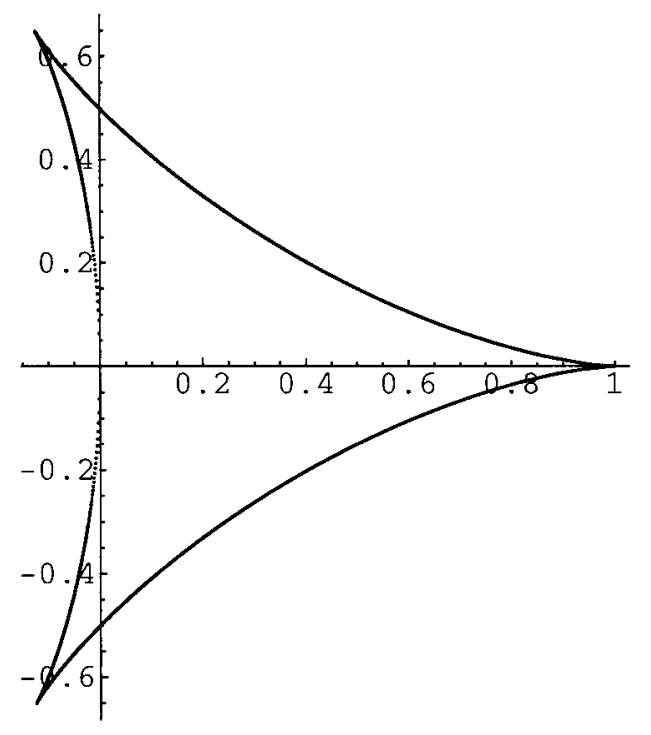

FigURE 1. Image of critical set for $f(z)=z^{2}+z-\bar{z}$

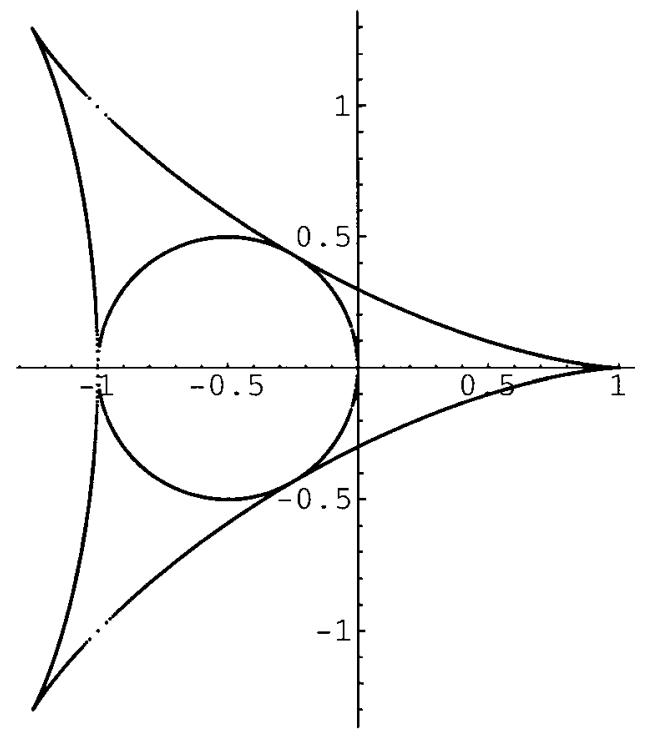

Figure 2. Preimage of $f(S)$ for $f(z)=z^{2}+z-\bar{z}$

Proof. Since $R$ is simply connected, there exist functions $g$ and $h$ holomorphic in $R$ such that $f=h+\bar{g}$ in $R$.

First, suppose that the zeros of $g^{\prime}$ are not isolated in $R$. By the identity theorem, we must have $g^{\prime} \equiv 0$ in $R$; thus $g$ is constant in our connected set $R$. Since int $S \neq \varnothing$, there exists a non-empty open disc $U \subseteq S$. Since $g^{\prime} \equiv 0, h^{\prime} \equiv 0$ in $U$. Since $h$ is constant in $U, h$ is constant in our connected set $R$. Hence, $f=h+\bar{g}$ 
is constant and maps $R$ to a single point; that point thus has infinite valence. The representation of $f$ follows trivially. Also note that $S=R$.

Now, suppose that the zeros of $g^{\prime}$ are isolated. We may then choose a nonempty open set $U \subseteq S$ such that $g^{\prime}(z) \neq 0$ for all $z \in U$. Then $\psi(z)=h^{\prime}(z) / g^{\prime}(z)$ is a holomorphic function on $U$ and $|\psi| \equiv 1$ on $U$. Take a non-empty closed disc $K \subset U$. By the maximum modulus principle, since $|\psi| \equiv 1$ on $K, \psi \equiv \lambda$ on $K$ for some unimodular constant $\lambda$. Moreover, $h^{\prime}-\lambda g^{\prime} \equiv 0$ on int $K$. Thus, $h^{\prime}-\lambda g^{\prime} \equiv 0$ on $R$ and $h^{\prime}(z)=\lambda g^{\prime}(z)$ for all $z \in R$. We thus have that $S=R$. Further, for some constant $\alpha \in \mathbb{C}, h=\alpha+\lambda g$. Choose $\tau$ such that $\tau^{2}=\lambda$. Then $f=h+\bar{g}=\alpha+2 \tau \operatorname{Re}(\tau g)=\alpha+\beta u$ in $R$, where $\beta=2 \tau$ and $u=\operatorname{Re}(\tau g)$ is a real-valued harmonic function in $R$. Choose $w_{0} \in f(R)$. Then we may choose $z_{0} \in R$ such that $f\left(z_{0}\right)=w_{0}$. Thus, $\alpha=w_{0}-\beta u\left(z_{0}\right)$. Consider $v(z)=u(z)-u\left(z_{0}\right)$. Since the zeros of a real-valued harmonic function are not isolated, $v$ has an infinite number of distinct zeros in $R$; hence $\operatorname{Val}\left(f, w_{0}\right)=\infty$.

Remark 4.8. The preceding lemma can also be found in Lyz92. If $f$ is harmonic in a simply connected, open set in $\mathbb{C}$ and if int $S \neq \varnothing$, then the preceding lemma implies that $f(R)$ is either a point or a subset of a line. Thus, $\operatorname{int}(f(S) \cup C(f))=\varnothing$. Moreover, if $w \in \mathbb{C} \backslash(f(S) \cup C(f))$, then $\operatorname{Val}(f, w)=0$.

Lemma 4.9. Let $f$ be harmonic in an open set $R \subseteq \mathbb{C}$. If int $S \neq \varnothing$, then there exists a point $w_{0} \in f(R)$ such that $\operatorname{Val}\left(f, w_{0}\right)=\infty$. Moreover, there exists an open, non-empty set $R_{1}$, with $R_{1} \subseteq S \subseteq R$, such that $f\left(R_{1}\right)$ is either a point or is a subset of a line.

Proof. Choose $z_{0} \in$ int $S$. Then we can find $\delta>0$ such that $B\left(z_{0}, \delta\right) \subseteq$ int $S$. Let $R_{1}=B\left(z_{0}, \delta\right)$. Then $R_{1}$ is a non-empty, simply connected, open set and we may apply Lemma 4.7 to $\left.f\right|_{R_{1}}$. The result follows.

Corollary 4.10. Let $f$ be harmonic in an open set $R \subseteq \mathbb{C}$. If $\operatorname{Val}(f, w)$ is finite for each $w \in \mathbb{C}$, then $\operatorname{int}(f(S) \cup C(f))=\varnothing$.

Proof. Since $f$ is $C^{\infty}$ on $R, f(S)$ has Lebesgue measure 0 by Sard's Theorem; hence, $f(S)$ has empty interior. By Lemma 4.9 since we assume that $\operatorname{Val}(f, w)$ is finite for each $w \in \mathbb{C}$, we must have int $S=\varnothing$. Since $S$ is relatively closed in $R$, we have that $S$ is nowhere dense in $R$. Since we have assumed that $\{w: \operatorname{Val}(f, w)=\infty\}=\varnothing$, $\operatorname{int}(C(f))=\varnothing$ by Theorem 3.9. The result follows from Remark 3.8 and Theorem 3.9 .

The results above relate to a theorem of T. J. Suffridge and J. W. Thompson [ST00. We need a definition before stating the theorem.

Definition 4.11 (Suffridge and Thompson). Assume $f$ is harmonic in $\{z: 0<$ $|z-\alpha|<r\}$ for some $r>0$. Define $\alpha$ to be a pole of $f$ provided $\lim _{z \rightarrow \alpha}|f(z)|=\infty$.

Theorem 4.12 (Suffridge and Thompson). Let $f$ be non-constant and harmonic, except for a finite number of poles, in a simply connected region $D$ in $\mathbb{C}$. Let $C$ be a Jordan curve contained within $D$ not passing through a pole. Let $\Omega$ be the region bounded by $C$. Let $X$ be the exceptional set of $f$ in $D$. Then the image space is partitioned into open components by $f(C \cup X)$ such that all values in a given component are assumed the same number of times in $\Omega$. 
The "exceptional set of $f$ " consists of points in $D$ where the dilatation of $f$ (defined as $\overline{f_{\bar{z}}} / f_{z}$ ) is unimodular. The proof of Theorem 4.12] in [ST00] assumes that the dilatation is analytic in $D$; this occurs if $S$ has no isolated critical points. When $S$ has no isolated critical points, $S=X$. As Example 4.14 below demonstrates, we must include the images of isolated critical points in the partitioning set.

We can correct this difficulty and extend Theorem 4.12 to the case of less restrictive conditions on $D$ and its boundary by using the partitioning results above, as follows:

Corollary 4.13. Let $f$ be harmonic in a bounded, open set $R \subset \mathbb{C}$. Suppose that $P=\operatorname{int}(\bar{R}) \backslash R$ is empty or consists of a finite number of poles of $f$. Suppose also that $f$ can be continuously extended to $\partial R \backslash P$. Then $f(S) \cup f(\partial R \backslash P)$ partitions $\mathbb{C}$ into regions of constant valence.

Proof. If we have a sequence $\left\{z_{n}\right\} \subset R$ approaching $z \in P$, then $f\left(z_{n}\right) \rightarrow \infty$. Thus, since $R$ is bounded, $C(f)=\left\{w: \exists\left\{z_{n}\right\} \subset R\right.$ such that $f\left(z_{n}\right) \rightarrow w$ and $z_{n} \rightarrow z_{0} \in$ $\partial R \backslash P\}$. Since $f$ can be continuously extended to $\partial R \backslash P, C(f)=f(\partial R \backslash P)$. Then apply Theorem 4.1

Example 4.14. $f(z)=\frac{1}{3} z^{3}-\frac{1}{2} \bar{z}^{2}$.

In this example, we will be looking at $f$ restricted to $R=B(0,1)$. This example illustrates why the image $(w=0)$ of the isolated critical point $(z=0)$ must be included in the partitioning set. We will look at $f$ as a function in $\mathbb{C}$ in Example 5.17 below.

Looking at $f$ in $B(0,1)$, calculations show that $S=\{0\}$ and that $C(f)=f(\{z$ : $|z|=1\}$ ). The partitioning set is graphed in Figure 3 (see Example [5.17 below) where the cusps are identified by numbers. Note that the partitioning set is the same set of points as in Example 5.17.

We will explicitly find the preimages of $w=0$ (Claim 2) and show that the valence is not constant in a sufficiently small neighborhood of $w=0$ (Claim 3.) It is helpful to rewrite $f$ as a function of $(x, y)$ where $z=x+i y$ :

$$
f(x, y)=\frac{1}{3} x^{3}-x y^{2}-\frac{1}{2} x^{2}+\frac{1}{2} y^{2}+i y\left(x^{2}-\frac{1}{3} y^{2}+x\right) .
$$

Claim 1: Let $y^{2}=3 x(x+1)$. Then $(x, y) \in B(0,1)$ iff $0 \leq x<1 / 4$.

Outline of proof. Note that $(x, y) \in R=B(0,1)$ iff $0 \leq x^{2}+y^{2}<1$. Using our condition on $y^{2}$, we require $-1 \leq 4 x^{2}+3 x-1<0$. We note that $\varphi(x)=4 x^{2}+3 x-1$ has zeros at $x=-1,1 / 4$ and attains its minimum at $x=-3 / 8$. So we require $-1<$ $x<1 / 4$. Now, $\varphi(0)=-1$ and $\varphi(-3 / 8)<-1$. We also need $y^{2}=3 x(x+1) \geq 0$ and that forces us to require $x \geq 0$.

Claim 2: $\operatorname{Val}\left(\left.f\right|_{R}, 0\right)=1$.

Outline of proof. To satisfy $\operatorname{Im} f(x, y)=0$, we must have $y=0$ or $y^{2}=3 x(x+1)$. We must also satisfy $R e f=0$. We can rewrite this as $(3-6 x) y^{2}+x^{2}(2 x-3)=0$. It is easy to check that this equation has no solutions when $x=\frac{1}{2}$. Thus, we must have $y^{2}=\left\{x^{2}(2 x-3)\right\} /(6 x-3)$. To get $f=0$ when $y=0$, we must have $x=0$ or $\frac{3}{2}$. To get $f=0$ when $y^{2}=3 x(x+1)$, we must have $x=\frac{3}{8}(-1 \pm \sqrt{5})$; neither value of $x$ satisfies Claim 1. Also note that the corresponding values of $y^{2}$ for both choices of $x$ are positive, so these correspond to preimages of 0 outside of $R$. So, $w=0$ has six distinct preimages in $\mathbb{C}$, but only one preimage $R=B(0,1)$; namely, $z=0$. 
Claim 3: Choose $|\epsilon|>0$, sufficiently small. Then $\operatorname{Val}\left(\left.f\right|_{R}, \epsilon\right)=2$.

Outline of proof. As in Claim 2 above, $\operatorname{Im} f=0$ requires $y=0$ or $y^{2}=3 x(x+1)$. We also require that $\operatorname{Re} f=\epsilon$.

First, let $y=0$. Then Re $f=\epsilon$ becomes $\varphi(x)=0$, where $\varphi(x)=\frac{1}{3} x^{3}-\frac{1}{2} x^{2}-\epsilon$ and $-1<x<1$ (to get a preimage in $R$ ). Now $\varphi^{\prime}(x)=0$ at $x=0,1 ; \varphi(0)=-\epsilon$ and $\varphi(1)=-\left(\epsilon+\frac{1}{6}\right)$. Note that $\varphi(-1)=-\left(\frac{5}{6}+\epsilon\right)$. Also $\varphi^{\prime \prime}(x)=0$ at $x=1 / 2$. An elementary calculus argument shows that we have no solutions for $\varphi(x)=0$ in $(-1,1)$ if $0<\epsilon<1 / 6$. Also, if $-\frac{1}{6}<\epsilon<0, \varphi$ has two distinct zeros in $(-1,1)$. Thus, for $|\epsilon|$ sufficiently small:

- If $\epsilon<0$, two distinct preimages of $w=\epsilon$ in $R$ on the real axis.

- If $\epsilon>0$, no preimages of $w=\epsilon$ in $R$ on the real axis.

Now, let $y^{2}=3 x(x+1)$. We may assume $y \neq 0$, since that case has already been handled. Then $\operatorname{Re} f=\epsilon$ becomes $\varphi(x)=0$, where $\varphi(x)=\frac{8}{3} x^{3}+2 x^{2}-\frac{3}{2} x+\epsilon$. By Claim 1, we also require $0 \leq x<1 / 4$. The zeros of $\varphi^{\prime}(x)$ are $x=1 / 4,-3 / 4$, while $\varphi^{\prime \prime}(x)=0$ at $x=-1 / 4$. Notice that $\varphi(0)=\epsilon, \varphi(1)=\frac{19}{6}+\epsilon>0$ for $|\epsilon|$ sufficiently small, and $\varphi(1 / 4)=-\frac{5}{24}+\epsilon<0$ for $|\epsilon|$ sufficiently small. Thus, for $|\epsilon|$ sufficiently small we have:

- If $\epsilon<0, \varphi(x)$ has no zeros in $[0,1 / 4)$. So, no preimages of $w=\epsilon$ in $R$ off the real axis.

- If $\epsilon>0, \varphi(x)$ has one zero in $[0,1 / 4)$; that root is not zero. Since $y^{2}=$ $3 x(x+1) \neq 0$ for $x \in(0,1 / 4)$, two distinct preimages of $w=\epsilon$ in $R$ off the real axis.

The claim follows by combining the two cases.

When does the partitioning set in the preceding corollary have empty interior?

Lemma 4.15. Let $f$ be harmonic in a bounded open set $R \subset \mathbb{C}$. Suppose that $P=\operatorname{int}(\bar{R}) \backslash R$ is empty or consists of a finite number of poles of $f$. Suppose also that $f$ has a $C^{1}$ extension to some open set $R_{1}$, where $R_{1} \supset \bar{R} \backslash P$. Then $f(S) \cup f(\partial R \backslash P)$ has empty interior.

Proof. The following proof was suggested by D. Sarason. Let $F$ denote our $C^{1}$ extension of $f$ to $R_{1}$ and $S_{F}$ denote the critical set of $F$. Let

$$
\begin{aligned}
& A=\partial R \backslash\left(S_{F} \cup P\right), \\
& B=S \cup\left(S_{F} \cap(\partial R \backslash P)\right) .
\end{aligned}
$$

Then $S \cup(\partial R \backslash P)=A \cup B$. If $F(A) \cup F(B)$ is a countable union of nowhere dense sets, then the result follows from the Baire Category Theorem.

We claim that $F(B)$ is a countable union of nowhere dense sets. By construction, $B \subseteq S_{F}$; hence $F(B) \subseteq F\left(S_{F}\right)$ and it is enough to show that $F\left(S_{F}\right)$ is a countable union of nowhere dense sets. $F\left(S_{F}\right)$ has measure 0 by Sard's Theorem. Since $S_{F}$ is closed in $R_{1}, S_{F}$ is a countable union of compact sets. Therefore $F\left(S_{F}\right)$ is a countable union of compact sets, each of which has measure 0 , hence each nowhere dense.

We also claim that $F(A)$ is a countable union of nowhere dense sets. We note that $F$ is a local homeomorphism at each $z \in A$, so for each $z \in A$, there exists $\epsilon>0$ such that $\left.F\right|_{B(z, 2 \epsilon)}$ is a homeomorphism. In particular, $B(z, 2 \epsilon) \cap\left(S_{F} \cup P\right)=\varnothing$. Then $\mathcal{C}=\{B(z, \epsilon): z \in A\}$ is an open cover of $A$. By Lindelöf's Theorem, $\mathcal{C}$ has a countable subcover, say $\left\{B\left(z_{j}, \epsilon_{j}\right): z_{j} \in A\right\}$. By construction, $K_{j}=$ 
$\overline{B\left(z_{j}, \epsilon_{j}\right) \cap A} \subseteq A$ and we see that $A=\bigcup K_{j}$. We note that $K_{j} \subseteq \partial R$ is compact and has empty interior. Since $F$ is a homeomorphism on $K_{j}, F\left(K_{j}\right)$ is nowhere dense. The claim follows by noting that $F(A)=\bigcup F\left(K_{j}\right)$.

\section{Application to harmonic mappings in the entire COMPlex plane}

If $f$ is harmonic in the entire complex plane, $C(f)=C(f, \infty)$. Also, we can write $f$ as $f=h+\bar{g}$ where $h$ and $g$ are entire (holomorphic) functions. This allows us to say more about the behavior of $f$ when $S$ has non-empty interior. In particular, we can apply Lemma 4.7 as follows:

Lemma 5.1. Let $f$ be harmonic on $\mathbb{C}$. If int $S \neq \varnothing$, then $f(\mathbb{C})$ is either a single point or a line. In either case, $S=\mathbb{C}$ and there exists a point $w_{0} \in \mathbb{C}$ such that $\operatorname{Val}\left(f, w_{0}\right)=\infty$.

Proof. Let $f=h+\bar{g}$, where $g$ and $h$ are entire (holomorphic) functions. The conclusions about $S$ and the existence of a point of infinite valence follow directly from Lemma 4.7 .

Now we return to the proof of Lemma 4.7 If the zeros of $g^{\prime}$ are not isolated, then $f(\mathbb{C})$ is a single point. If the zeros of $g^{\prime}$ are isolated, $g$ is a non-constant entire function and there exists a unimodular constant $\lambda$ such that $h^{\prime} \equiv \lambda g^{\prime}$. Choosing $\tau$ such that $\tau^{2}=\lambda$ and choosing $z_{0}$ such that $f\left(z_{0}\right)=w_{0}$, we have $f=h+\bar{g}=$ $\alpha+2 \tau \operatorname{Re}(\tau g)$ where $\alpha=w_{0}-2 \tau \operatorname{Re}\left(\tau g\left(z_{0}\right)\right)$.

Since $\tau$ is non-zero and $g$ is a non-constant entire function, $\varphi(z)=\operatorname{Re}(\tau g(z))$ is a non-constant, real harmonic function in the entire plane. By Liouville's Theorem, $\varphi(z)$ can be bounded neither above nor below. Noting that the range of $\varphi$ is connected, we see that the range of $\varphi$ is the entire real axis. Hence $f(\mathbb{C})$ is a line when the zeros of $g^{\prime}$ are isolated.

Lemma 5.2. Let $f$ be harmonic on $\mathbb{C}$. Suppose that int $S \neq \varnothing$. Then $\operatorname{int}(f(S) \cup$ $C(f, \infty))=\varnothing$. Moreover, if $w \in \mathbb{C} \backslash(f(S) \cup C(f, \infty))$, then $\operatorname{Val}(f, w)=0$.

Proof. By Lemma 5.1, $S=\mathbb{C}$ and $f(\mathbb{C})$ is either a point or a line (each is a closed set with empty interior in $\mathbb{C})$. Clearly, $C(f, \infty) \subseteq f(S)=f(\mathbb{C})$. Hence, $f(S) \cup C(f, \infty)$ has empty interior. Also, if $w \in \mathbb{C} \backslash(f(S) \cup C(f, \infty))$, then $w \in \mathbb{C} \backslash f(\mathbb{C})$ and thus $\operatorname{Val}(f, w)=0$.

We also can say more about when the partitioning set $f(S) \cup C(f, \infty)$ has empty interior. We first recall a result of Wilmshurst Wil94:

Theorem 5.3 (Wilmshurst). Let $f(z)=\overline{g(z)}+h(z)$ be a function harmonic in the (entire) complex plane. If $\lim _{z \rightarrow \infty} f(z)=\infty$, then $f$ has finitely many zeros.

Note that $\lim _{z \rightarrow \infty} f(z)=\infty$ implies that $\operatorname{Val}(f, w)$ is finite for each $w$ (note that this does not imply that $f$ has finite valence). We also note that if $\lim _{z \rightarrow \infty} f(z)=$ $\infty$, then $C(f, \infty)=\varnothing$. Since $f(S)$ has empty interior by Sard's Theorem, we have that $f(S) \cup C(f, \infty)=f(S)$ has empty interior. In particular,

Corollary 5.4. If $f$ is a harmonic mapping on $\mathbb{C}$ such that $\lim _{z \rightarrow \infty} f(z)=\infty$, then $f(S)$ partitions $\mathbb{C}$ into non-empty regions of constant valence.

Corollary 5.4 obviously applies if $f(z)=p(z)-\overline{q(z)}$, where $p$ and $q$ are polynomials in $z$ of different degree. D. Bshouty, W. Hengartner and M. Naghibi-Beidokhti in BHN99] use approximation theory to show that there exist harmonic mappings 
on $\mathbb{C}$ which are not polynomials such that $\lim _{z \rightarrow \infty} f(z)=\infty$. An explicit example of such a function is due to D. Sarason; consider $f(z)=\operatorname{Re}\left(e^{-z^{2}}\right)+z$. Calculations also show that $f$ is onto $\mathbb{C}$. While $\operatorname{Val}(f, w)$ is finite for each $w, \operatorname{Val}(f)$ is infinite.

We will show that $f(S) \cup C(f, \infty)$ has empty interior when $f$ is a harmonic polynomial, even when Corollary 5.4 does not apply. To do so, we need to state several results. We start with a result found in Wil98:

Theorem 5.5 (Wilmshurst). Let a function $f$ be harmonic in some domain $D$ and have a sequence of distinct zeros $\left\{z_{m}\right\}$ converging to some point $z^{*}$ in $D$. Then $f(z) \equiv 0$ on some simple analytic arc containing $z^{*}$ as an interior point. Further, there are at most finitely many such arcs unless $f(z) \equiv 0$ in $D$.

We also note two forms of Bézout's Theorem found in Wilmshurst Wil98:

Theorem 5.6 (Wilmshurst: Bézout's Theorem). Let $A$ and $B$ be relatively prime polynomials in the real variables $x$ and $y$ with real coefficients, and let $\operatorname{deg} A=n$ and $\operatorname{deg} B=m$. Then the two algebraic curves $A(x, y)=0$ and $B(x, y)=0$ have at most mn points in common.

Theorem 5.7 (Wilmshurst: Alternate form of Bézout's Theorem). Let $A$ and $B$ be polynomials in the real variables $x$ and $y$ with real coefficients. If $\operatorname{deg} A=n$ and $\operatorname{deg} B=m$, then either $A$ and $B$ have at most $m n$ common zeros or have infinitely many common zeros.

Remark 5.8. Bézout's Theorem also provides information about a real algebraic curve in the plane. Let $P(x, y)$ be a polynomial in the real variables $x$ and $y$ with real coefficients. There exist irreducible polynomials in $x$ and $y$ with real coefficients, say $A_{1}(x, y), \ldots, A_{k}(x, y)$, such that $P=A_{1}^{n_{1}} \ldots A_{k}^{n_{k}}$. Let $V=\{(x, y): P(x, y)=0\}$. Then $V=\bigcup_{j=1}^{k} V_{j}$, where $V_{j}=\left\{(x, y): A_{j}(x, y)=0\right\}$. Let $D_{j}=\{(x, y)$ : $\left.\partial A_{j} / \partial y=0\right\}$. Since the $A_{j}$ are relatively prime, if $i \neq j$, then $V_{i} \cap V_{j}$ is finite by Theorem 5.6. If $\partial A_{j} / \partial y \equiv 0$, then $V_{j}$ consists of a finite number (possibly zero) of vertical lines. If $\partial A_{j} / \partial y$ is not identically zero, then $V_{j} \cap D_{j}$ is finite by Theorem 5.6 since $A_{j}$ is irreducible. In that case, we can divide the plane into a finite number of vertical strips such that $\partial A_{j} / \partial y \neq 0$ for points in $V_{j}$ in the interior of a given vertical strip. By the implicit function theorem, using analytic continuation, we see that $V_{j}$ consists of a finite number (possibly zero) of non-intersecting curves in the interior of a given vertical strip [Smi71, pp. 249-253] and possibly a finite number of vertical lines. If $V_{j}$ contains a closed loop, we see that $V_{j}$ must contain a simple loop. A closed loop in $V$ is either a closed loop in one of the $V_{j}$ or is formed by joining arcs in $V$ terminating at points in $V_{i} \cap V_{j}$ where $i \neq j$. Since there are finitely many points in the $V_{i} \cap V_{j}$, we see that if $V$ contains a closed loop, then $V$ contains a simple loop.

We also need a fact about harmonic polynomials from [BC55]:

Lemma 5.9 (M. Brelot and G. Choquet). If a real harmonic polynomial $p$ in two variables has a non-constant factor $q$, then the zeros of $q$ are not isolated.

Lemma 5.10. Let $f$ be a harmonic polynomial. Let $\gamma$ be a closed loop in $S$. If $S$ has empty interior, then $f$ cannot be constant on $\gamma$.

Proof. Since $S$ has empty interior, we can view $S$ as a real algebraic curve. By Remark 5.8 if $S$ contains a closed loop $\gamma$, we can find a simple loop in $\gamma$. Without 
loss of generality, we may suppose that $\gamma$ is a Jordan curve. Then $\gamma$ encloses some bounded, connected region, say $R$, by the Jordan curve theorem.

Assume that $f$ is constant on $\gamma$. Let $u=\operatorname{Re} f$ and $v=\operatorname{Im} f$. Then $u$ and $v$ are real harmonic functions in $\mathbb{C}$. Since $f$ is constant on $\gamma=\partial R$, so are $u$ and $v$. Hence, $u$ must be constant in $R$ by the minimum and maximum principles for real harmonic functions. Similarly, $v$ is constant in $R$. Thus, $f$ is constant in $R$ and hence in $\mathbb{C}$. We have $S=\mathbb{C}$, a contradiction.

The preceding proof uses an argument similar to that used by Wilmshurst (pages 42-43 in Wil94]) to show that the zeros of a harmonic polynomial are isolated under certain conditions.

Corollary 5.11. If $f$ is a harmonic polynomial, then $\operatorname{int}(f(S) \cup C(f, \infty))=\varnothing$.

Proof. The result is obvious if $f$ is constant, so we will assume that $f$ is a nonconstant harmonic polynomial. Following Wilmshurst Wil98, finding all $z$ such that $f(z)-w=0$ is equivalent to finding the common zeros of $\operatorname{Re}(f-w)$ and $\operatorname{Im}(f-w)$ in $\mathbb{R}^{2}$, identifying $\mathbb{C}$ with $\mathbb{R}^{2}$ in the obvious way.

Let $n=\operatorname{Max}\{\operatorname{deg}(\operatorname{Re} f), \operatorname{deg}(\operatorname{Im} f)\}$. By Theorem [5.7 Re $(f-w)$ and $\operatorname{Im}(f-w)$ have either at most $n^{2}$ common zeros or have infinitely many common zeros. Hence $\operatorname{Val}(f) \leq n^{2}$ or there exists $w_{0} \in \mathbb{C}$ such that $\operatorname{Val}\left(f, w_{0}\right)=\infty$.

If $\operatorname{Val}(f)$ is finite, the result follows from Corollary 4.10, So we may suppose that there exists $w_{0} \in \mathbb{C}$ such that $\operatorname{Val}\left(f, w_{0}\right)=\infty$. If $S$ has non-empty interior, we are in the simple case where $f$ maps $\mathbb{C}$ to a point or a line by Lemma 5.1, and the result follows. So, suppose also that $S$ has empty interior. Since $S$ is closed in $\mathbb{C}$, we also have that $S$ is nowhere dense. Since $f$ is harmonic, $f(S)$ has empty interior by Sard's Theorem. We want to show that $W_{\infty}=\{w: \operatorname{Val}(f, w)=\infty\}$ is nowhere dense. If that is true, the result follows from Theorem 3.9 and Remark 3.8. We will show that $W_{\infty}$ is finite.

Since $\operatorname{Val}\left(f, w_{0}\right)=\infty$, there are infinitely many distinct values of $z$ such that $f(z)=w_{0}$. By Bézout's Theorem (Theorem 5.6), $\operatorname{Re}\left(f-w_{0}\right)$ and $\operatorname{Im}\left(f-w_{0}\right)$ have a non-trivial common factor. Choose $z_{0}$ such that $f\left(z_{0}\right)=w_{0}$. By the BrelotChoquet Lemma (Lemma 5.9), $z_{0}$ is not an isolated zero of $f(z)-w_{0}$. Hence, by Theorem 5.5. there exists a simple analytic arc $\gamma$ such that $\left.f\right|_{\gamma} \equiv w_{0}$ and such that $z_{0}$ is in the interior of $\gamma$. Since $f$ is not locally 1-1 at any point in the interior of $\gamma$, int $\gamma \subseteq S$. Further, we can apply Theorem 5.5 to each of the endpoints of $\gamma$ to see that $\gamma \subseteq S$. If $\gamma$ contains a closed loop, then $S$ has non-empty interior by Lemma 5.10, a contradiction. So we may suppose that $\gamma$ does not contain a closed loop. Thus, for each $w \in W_{\infty}$, we can find a simple curve $\gamma$ in $S$ such that $\left.f\right|_{\gamma} \equiv w$.

Since $f$ is a harmonic polynomial, $J_{f}(z)$ can be viewed as a polynomial in $\mathbb{R}^{2}$ and Remark 5.8 applies to $S$. Moreover, if we extend $\gamma$ by repeated application of Theorem [5.5, we see that $\gamma$ extends to an unbounded curve such that $\left.f\right|_{\gamma} \equiv w_{0}$. Otherwise, if our extensions of $\gamma$ have a finite limit point $z_{0}$, by continuity, $f\left(z_{0}\right)=$ $w_{0}$ and we can extend $\gamma$ through $z_{0}$, a contradiction. From Remark [5.8 we see that $\gamma$ can be extended to a simple, unbounded curve in $S$. From Remark 5.8, we see that $S$ contains at most finitely many simple, unbounded curves. Hence $W_{\infty}$ contains a finite number of points and the result follows.

Example 4.4 is an example of a harmonic polynomial with int $S=\varnothing$ and a point with infinite valence. In this example, the preimages of this point are non-isolated and lie on a curve (the line $\operatorname{Re} z=\operatorname{Im} z$ ). 
The situation is not so nice when $f$ is not a harmonic polynomial. We will restrict our attention to the case $R=\mathbb{C}$. For example, if $f$ is an entire (holomorphic) transcendental function, $C(f, \infty)$ is the complex plane by Picard's Theorem. The monograph of M. Balk [Bal91] concerns the theory of polyanalytic and polyentire functions; results similar to Picard's Theorem are given for polyentire functions (see pages 107-109.)

Recall that $\varphi(z)$ is a polyanalytic function of order $n$ if it can be written as $\varphi(z)=\Sigma_{k=0}^{n-1} a_{k}(z) \bar{z}^{k}$ where each $a_{k}(z)$ is a holomorphic function of $z$. If each $a_{k}(z)$ is entire, then $\varphi$ is polyentire. Let $h$ and $g$ be holomorphic in some region $R$ and let $f=h+\bar{g}$. Then the harmonic function $f$ can be thought of as a polyanalytic function (of countable order if $g$ is transcendental). If $g(z)=\sum_{k=0}^{m} b_{k} z^{k}$ is a nonconstant polynomial, we can think of $f$ as a polyanalytic function of finite order by putting $a_{0}(z)=h(z)+\overline{b_{0}}$ and $a_{k}(z) \equiv \overline{b_{k}}$ for $1 \leq k \leq \operatorname{deg} g$.

Let $P(z, \bar{z})$ denote a polynomial in $z$ and $\bar{z}$. Note that $P$ can include terms of the form $z^{n} \bar{z}^{m}$. Consider the following result from [Bal91] about polyentire functions:

Theorem 5.12 (Balk). Let $E(z)$ be an entire transcendental analytic function, and let $P(z, \bar{z})$ be an arbitrary polyanalytic but not analytic polynomial. Then the polyentire function $F(z)=E(z)+P(z, \bar{z})$ assumes in the complex plane $\mathbb{C}$ every complex value $A$ (without any exceptions!) The set $M(F ; A)$ of all $A$-points of the polyentire function $F(z)$ is for every $A$ unbounded and discrete in $\mathbb{C}$.

If $f=h+\bar{g}$ is harmonic in $\mathbb{C}$, the preceding result tells us that $C(f, \infty)=\mathbb{C}$ if $h$ is transcendental and $g$ a non-constant polynomial. Picard's Theorem takes care of the case when $h$ is transcendental and $g$ a constant. This gives us a necessary condition for $f(S) \cup C(f, \infty)$ to have empty interior:

Corollary 5.13. Let $f=h+\bar{g}$ be harmonic in $\mathbb{C}$, where $h$ and $g$ are entire. If $f(S) \cup C(f, \infty)$ has empty interior, then one of the following holds: (i) $h$ and $g$ are both polynomials in $z$ or (ii) $h$ and $g$ are both entire transcendental functions in $z$.

Does the converse hold? Corollary 5.11 shows that the converse holds for harmonic polynomials. We have seen some examples where $h$ and $g$ are both transcendental and $f(S) \cup C(f, \infty)$ has empty interior (see Example 4.2 and the comments after Corollary [5.4). Both examples are of the form $f(z)=z+\operatorname{Re} h(z)$ where $h$ is an entire transcendental function.

5.1. Joining regions for $f$ harmonic. Let $f$ be a light harmonic function in $\mathbb{C}$. Suppose that $R_{1}$ and $R_{2}$ are two distinct components of $\mathbb{C} \backslash f^{-1}(f(S) \cup C(f, \infty))$. Suppose that that $R_{1}$ and $R_{2}$ share a common boundary arc. Let $f\left(R_{1}\right)=\Omega_{1}$ and $f\left(R_{2}\right)=\Omega_{2}$. If $\Omega_{1}$ and $\Omega_{2}$ are each simply connected, then $f$ is univalent in $R_{1}$ and in $R_{2}$ by Theorem 3.13. Can we join $R_{1}$ and $R_{2}$ along the interior of the shared boundary arc to get a new region where $f$ is univalent?

If the regions we want to combine all lie in a convex domain, a partial answer is given in [Wil94]:

Theorem 5.14 (Sheil-Small). If $g(z)$ is an analytic function in the convex domain $D$ and $\left|g^{\prime}(z)\right|<1$ in $D$, then $\bar{z}+g(z)$ is univalent on $D$.

Let $h(z)$ be holomorphic in some convex domain $D$ and suppose that $\left|h^{\prime}(z)\right|<1$ in $D$. It is easy to see that this result also holds for $f(z)=h(z)+\lambda \bar{z}$ where $\lambda$ is a unimodular constant (apply the theorem to $f_{1}(z)=\bar{\lambda} f(z)$ ). 
We can apply this result to each of the bounded components of $\mathbb{C} \backslash S$ in Example 5.21 below to show that $f$ is univalent on each of these two bounded components. However, this result won't help us in the case that a bounded component of $\mathbb{C} \backslash S$ is not convex; consider, for example, the critical set in Example 5.22 below. Also, this result will not help us find univalent regions in the unbounded component of $\mathbb{C} \backslash S$ nor will it help us with more complicated harmonic functions.

What do the results in the preceding sections tell us about finding univalent regions? By identifying $\mathbb{C}$ with $\mathbb{R}^{2}$ in the obvious way, we may apply the preceding results.

Lemma 5.15. Let $f$ be a light harmonic function in $\mathbb{C}$. Let $R$ be a bounded component of $\mathbb{C} \backslash f^{-1}(f(S) \cup C(f, \infty))$. Choose a component $\Omega \subseteq \mathbb{C} \backslash(f(S) \cup C(f, \infty))$ such that $f(R)=\Omega$. Suppose that $w_{0}$ is an isolated point in $\partial \Omega$. If $f^{-1}\left(w_{0}\right) \cap$ $\partial R \cap S=\varnothing$, then $w_{0}$ has exactly $\operatorname{Val}\left(\left.f\right|_{R}, \Omega\right)$ distinct preimages in $\partial R$. These preimages are isolated points in $\partial R$.

Proof. Note that $\Omega$ exists by Theorem 3.10. We first check that $\zeta \in f^{-1}\left(w_{0}\right) \cap \partial R$ is isolated in $\partial R$. Suppose not. We first note that $\zeta$ is isolated in $f^{-1}\left(w_{0}\right)$. Otherwise, by Theorem 5.5 there exists a non-degenerate arc $\gamma$ such that $f$ is constant on $\gamma$. This contradicts $f$ being light; hence $\zeta$ is isolated in $f^{-1}\left(w_{0}\right)$. By Lemma 3.19. $f(\partial R)=\partial \Omega$. Hence if $\zeta$ is not isolated in $\partial R$, then there exists $\left\{\zeta_{n}\right\} \subseteq \partial R \backslash\{\zeta\}$ such that $\zeta_{n} \rightarrow \zeta$. Since $\zeta$ is isolated in $f^{-1}\left(w_{0}\right), f\left(\zeta_{n}\right) \neq w_{0}$ for $n$ sufficiently large. Since $\left\{f\left(\zeta_{n}\right)\right\} \subseteq \partial \Omega$ and $f\left(\zeta_{n}\right) \rightarrow w_{0}$ by continuity, $w_{0}$ is not isolated in $\partial \Omega$, a contradiction.

We need to check that the conditions in Theorem 3.21 are satisfied. Since $R$ is bounded, $C\left(\left.f\right|_{R}, \infty\right)=\varnothing$ and (i) holds. By assumption, (ii) holds. Since $R$ is bounded and $f$ is harmonic in $\mathbb{C}$, we can look at a sequence in $\Omega$ converging to $w_{0}$ and look at the corresponding sequence of preimages in $R$ to see that (iii) holds. Since the preimage points of $w_{0}$ are isolated in $\partial R$, (iv) also holds. The claim concerning the number of preimages follows from Theorem 3.21.

Corollary 5.16. Let $f$ be a light harmonic function in $\mathbb{C}$. Let $\Omega$ be a component of $\mathbb{C} \backslash(f(S) \cup C(f, \infty))$. Suppose that $($ int $\bar{\Omega}) \backslash \Omega$ consists of a finite number of points. Suppose that there exists a bounded component, say $R$, of $\mathbb{C} \backslash f^{-1}(f(S) \cup C(f, \infty))$ such that $f(R)=\Omega$. Suppose that every point in (int $\bar{R}) \backslash R$ is mapped to $($ int $\bar{\Omega}) \backslash \Omega$. If $($ int $\bar{R}) \backslash R$ contains no point in $S$, then $f$ is $\operatorname{Val}\left(\left.f\right|_{R}, \Omega\right): 1$ in int $\bar{R}$. Moreover, if int $\bar{\Omega}$ is simply connected, then $f$ is univalent in int $\bar{R}$.

Proof. Since $R$ is bounded, we can apply Lemma 5.15 to $R$ to get that each of the isolated points in $\partial \Omega$ has $\operatorname{Val}\left(\left.f\right|_{R}, \Omega\right)$ preimages in $\partial R$, which are puncture points in $R$. Thus, each point in int $\bar{\Omega}$ has exactly $\operatorname{Val}\left(\left.f\right|_{R}, \Omega\right)$ distinct preimages in int $\bar{R}$, the first claim. Since $S \cap i n t \bar{R}=\varnothing$, we see that int $\bar{R}$ is a $\operatorname{Val}\left(\left.f\right|_{R}, \Omega\right)$-fold covering of int $\bar{\Omega}$ (see the proof of Theorem 3.13) The second claim follows from Theorem 3.12 .

Example 5.17. $f(z)=\frac{1}{3} z^{3}-\frac{1}{2} \bar{z}^{2}$.

We looked at this function restricted to $\{z:|z|<1\}$ in Example 4.14above. We will now look at $f$ as a function in $\mathbb{C}$. Calculations show that $S=\{z:|z|=1\} \cup\{0\}$ and that $C(f, \infty)=\varnothing$. The partitioning set for $f$ will be $f(S)$, which is graphed in Figure 3 We can select a few points in each region of the partition and ask Mathematica to find the preimages. $\operatorname{Val}(f, w)=3$ in the unbounded component 


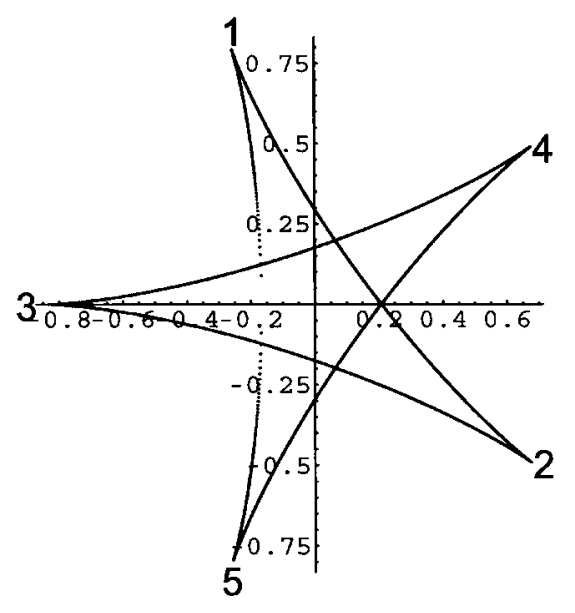

Figure 3. Image of critical set for $f(z)=\frac{1}{3} z^{3}-\frac{1}{2} \bar{z}^{2}$, with labels. Note that $0 \in f(S)$.

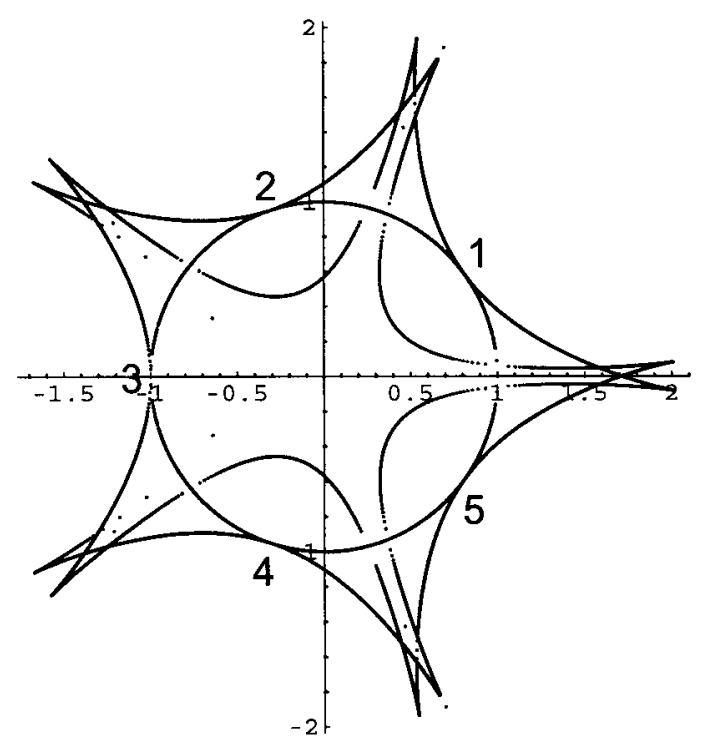

Figure 4. Preimage of $f(S)$ for $f(z)=\frac{1}{3} z^{3}-\frac{1}{2} \bar{z}^{2}$

of $\mathbb{C} \backslash f(S)$. In each "point of the star" region, $\operatorname{Val}(f, w)=5 . \operatorname{Val}(f, w)=7$ in the "center of the star" region (recall that the origin is in $f(S)$ and is not in this region). We have numbered the cusps in $f(S)$ in Figure 3 The critical points mapped to a given cusp are labeled with the same number in Figure 4 .

Recall that the origin is an isolated critical point of $f$. From the calculations in Example 4.14, we see that the origin has six distinct preimages. From Figure 4, we see that the preimages other than the origin lie in regions in the arms of the "star" (one in each such region). From the calculation in Example4.14, we see that $f$ is 2:1 in the component with the origin as a puncture point. Hence $f$ must be univalent 
in each of the five other components which have $f^{-1}(0)$ as a puncture point. It is not completely obvious from the figure that the preimage of the origin is an isolated point in each of the regions, because the Mathematica routines plot single points. However, in a region where $f^{-1}(0)$ is clearly a puncture point (for example, the arm in the lower right quadrant), this serves as an example of Corollary 5.16. We can fill in the puncture point not in $S$ in each such component of the preimage to get a simply connected region where $f$ is univalent.

Theorem 5.18. Let $f$ be a light, harmonic function in $\mathbb{C}$. Let $R_{1}$ and $R_{2}$ be disjoint bounded components of $\mathbb{C} \backslash f^{-1}(f(S) \cup C(f, \infty))$. Suppose that $R_{1}$ and $R_{2}$ each have at most a finite number of puncture points. Suppose that for each component $\Omega \subseteq \mathbb{C} \backslash(f(S) \cup C(f, \infty))$, $\partial \Omega \cap$ int $\bar{\Omega}$ consists of a finite number of points. Suppose that $f$ is univalent on $R_{1}$ and on $R_{2}$. Let $\gamma$ be a non-degenerate, simple arc in $\partial R_{1} \cap \partial R_{2}$. Suppose that $f($ int $\gamma)$ is a simple arc. If $S \cap$ int $\gamma=\varnothing$, then

(1) $f\left(R_{1}\right)$ and $f\left(R_{2}\right)$ are distinct components of $\mathbb{C} \backslash(f(S) \cup C(f, \infty))$.

(2) $f$ is a univalent on $R_{1} \cup R_{2} \cup$ int $\gamma$.

Proof. This follows from Theorem 3.23 by associating $\mathbb{R}^{2}$ and $\mathbb{C}$ in the usual way.

Remark 5.19. Note that if int $\gamma$ contains a non-degenerate subarc in $S$, then there exists $z_{0} \in$ int $\gamma$ such that $f_{z_{0}} \sim z, \bar{z}$ (see the proof of Theorem 6.7.) Thus, $f\left(R_{1}\right) \cap f\left(R_{2}\right) \neq \varnothing$. By Theorem 3.10 $f\left(R_{1}\right)=f\left(R_{2}\right)$.

Extending these ideas to joining more than two adjacent regions is more difficult. Applying these results requires assumptions as to whether pairs of components (both in the domain and in the range) have boundaries intersecting in more than one simple arc. Even if we join two regions, it could be the case that the images of the two regions meet in more than one simple arc; joining along more than one of these arcs could cause trouble.

If we have components as in Theorem 5.18, we can fill in puncture points if the conditions in Corollary [5.16 are satisfied to get a simply connected region of univalence. The following theorem of M. Ortel and W. Smith [OS86] will then help us join more than two univalent components, provided that the combined components in both the domain and range are simply connected.

Theorem 5.20 (Ortel and Smith). If $\Omega_{1}$ and $\Omega_{2}$ are open, connected, and simply connected subsets of the complex plane, $f: \Omega_{1} \rightarrow \Omega_{2}$ is continuous, surjective, and locally univalent, $N \in\{1,2,3, \ldots\}$ and $\# f^{-1}(w) \in\{1, N\}$ for all $w \in \Omega_{2}$, then $f$ is univalent.

For example, suppose that we have three disjoint simply connected components of $\mathbb{C} \backslash f^{-1}(f(S) \cup C(f, \infty))$ where $f$ is univalent and the image of each component is simply connected. Suppose also that the conditions of Theorem 5.18 hold pairwise. We thus have either two or three distinct image components. $f$ is univalent in the interior of each of the two boundary arcs used for joining regions. By construction, at least one of the image regions has preimages in only one of the original components. Thus, a point in the image of the combined image region must have either one or two preimages in the combined region. Univalence in the combined region follows from the theorem of Ortel and Smith, provided that we show that the combined image region is simply connected (see Examples 5.21 and 5.22). 


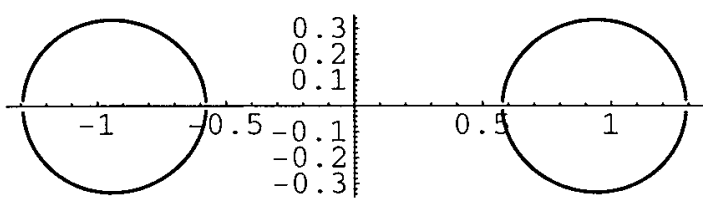

Figure 5. Critical set for $f(z)=-\frac{1}{2} z^{3}+\frac{3}{2} z-\bar{z}$

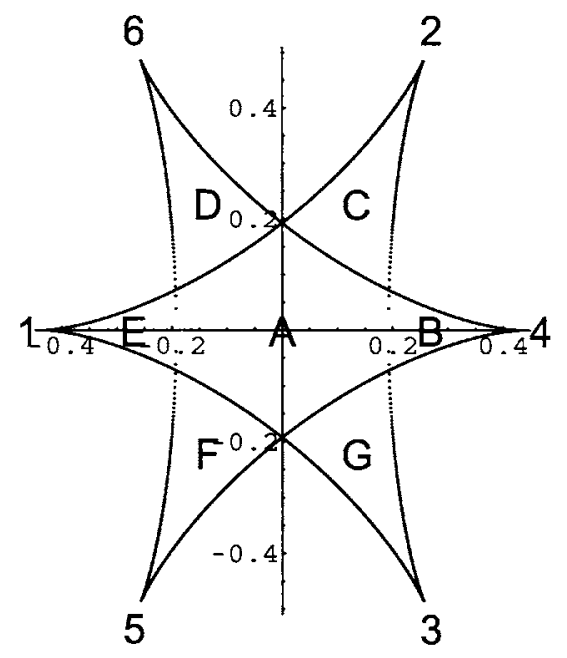

Figure 6. Image of critical set for $f(z)=-\frac{1}{2} z^{3}+\frac{3}{2} z-\bar{z}$, with labels

Example 5.21. $f(z)=-\frac{1}{2} z^{3}+\frac{3}{2} z-\bar{z}$.

Clearly, $C(f, \infty)=\varnothing$; thus the partitioning set is $f(S)$. The critical set of $f$ is graphed in Figure 5 it consists of two disjoint, closed curves. Figure 6 shows $f(S)$, with the cusps numbered and regions labeled in upper case letters. Using Mathematica to find preimages of a few points in each component of the partition, we see that $\operatorname{Val}(f, w)=3$ on the unbounded component, $\operatorname{Val}(f, w)=5$ on the tips of the star, and $\operatorname{Val}(f, w)=7$ in the "center" of the star.

Notice that each of the image regions is simply connected; hence we expect $f$ to be univalent on each of the corresponding preimage regions by Theorem 3.13 This is consistent with the results for finding the preimages of a point in each component of $\mathbb{C} \backslash f(S)$ using Mathematica.

The preimages of the cusps on the critical set are labeled in Figure 7 with the same numbers used in Figure 6. We have labeled the preimage components corresponding to a given bounded component of $\mathbb{C} \backslash f(S)$ with the corresponding letter.

As expected from Theorem 5.18, we can join components sharing a common arc with interior off $S$ to get a larger region of univalence. From Figure 7 it is apparent that we can continue joining such bounded components in the unbounded component of $\mathbb{C} \backslash S$ until we reach $S$. The combined regions in the image and in the domain appear to be simply connected, so univalence in the combined region should follow from the theorem of Ortel and Smith (Theorem 5.20). 


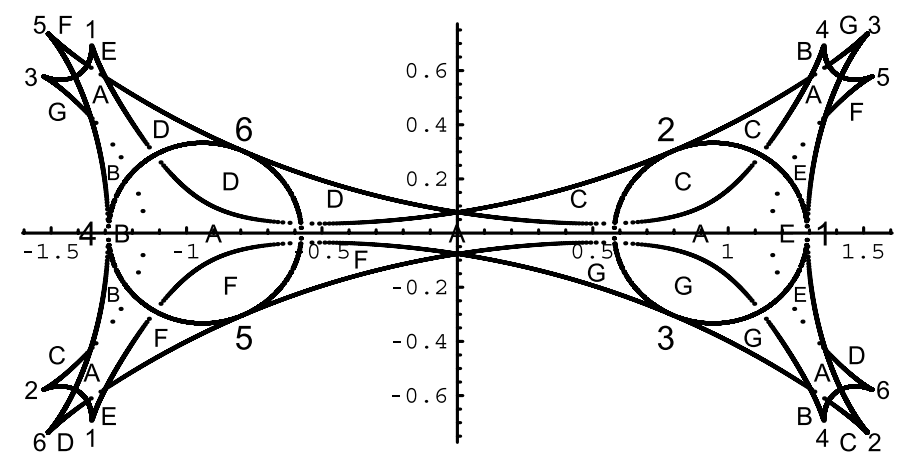

FiguRE 7. Preimage of $f(S)$ for $f(z)=-\frac{1}{2} z^{3}+\frac{3}{2} z-\bar{z}$

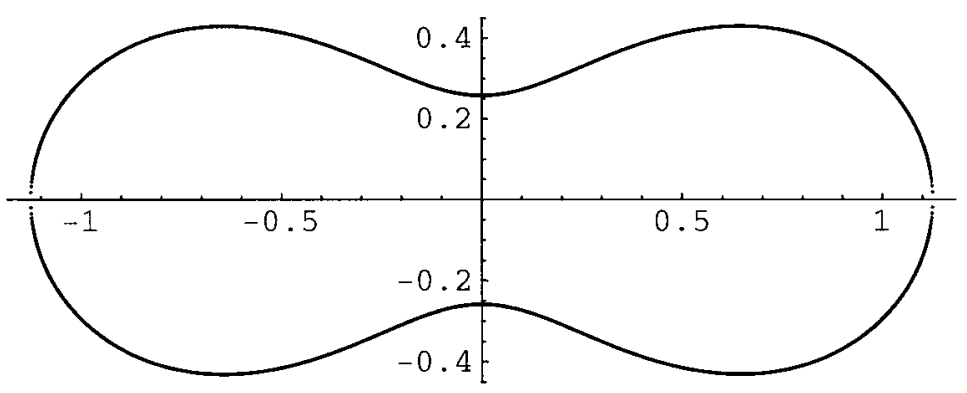

FIgURE 8. Critical set for $f(z)=-\frac{1}{2} z^{3}+\frac{9}{10} z-\bar{z}$

Example 5.22. $f(z)=-\frac{1}{2} z^{3}+\frac{9}{10} z-\bar{z}$.

$C(f, \infty)=\varnothing$; thus the partitioning set is $f(S)$. The critical set of $f$ is graphed in Figure 8, it looks like a dumbbell. Figure 9 shows $f(S)$, with the cusps numbered and two regions labeled in upper case letters. Using Mathematica to find preimages of a few points in each component of the partition, we see that $\operatorname{Val}(f, w)=3$ on the unbounded component and increases by two each time we cross an arc in $f(S)$ into a component containing the tangent to the arc. The components with maximum valence (7) are bisected by the imaginary axis.

Notice that each of the image regions appears to be simply connected; hence we expect $f$ to be univalent on each of the corresponding preimage regions by Theorem 3.13 .

In Figure 10, we label the regions of the partition of the domain inside the bounded component of $\mathbb{C} \backslash S$ which correspond to our two labeled image regions. Note that if we combine the three regions meeting at the point numbered 3 (a preimage of the cusp numbered 3 in Figure 9), we run into trouble. Let's call that point $z_{0}$. Note that $z_{0}$ is the only shared boundary point in $S$ for the three regions and is not included in the combined region. We note that $f\left(z_{0}\right)$ is a puncture point in the combined region, so the theorem of Ortel and Smith (Theorem5.20) cannot be applied to claim that $f$ is univalent on the combined region in the domain.

In Figure 10, we only labeled the preimages in $S$ of cusps. 


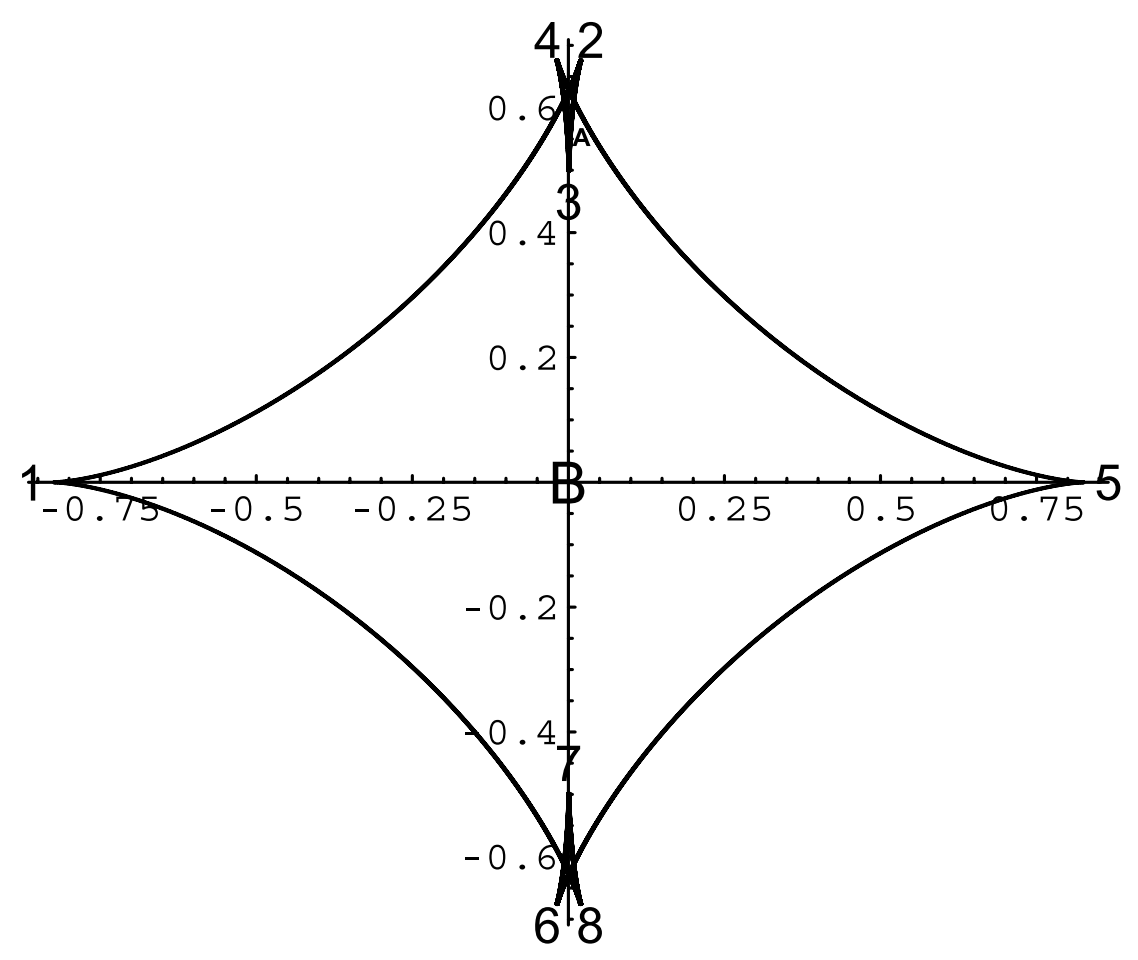

FiguRE 9. Image of critical set for $f(z)=-\frac{1}{2} z^{3}+\frac{9}{10} z-\bar{z}$, with labels

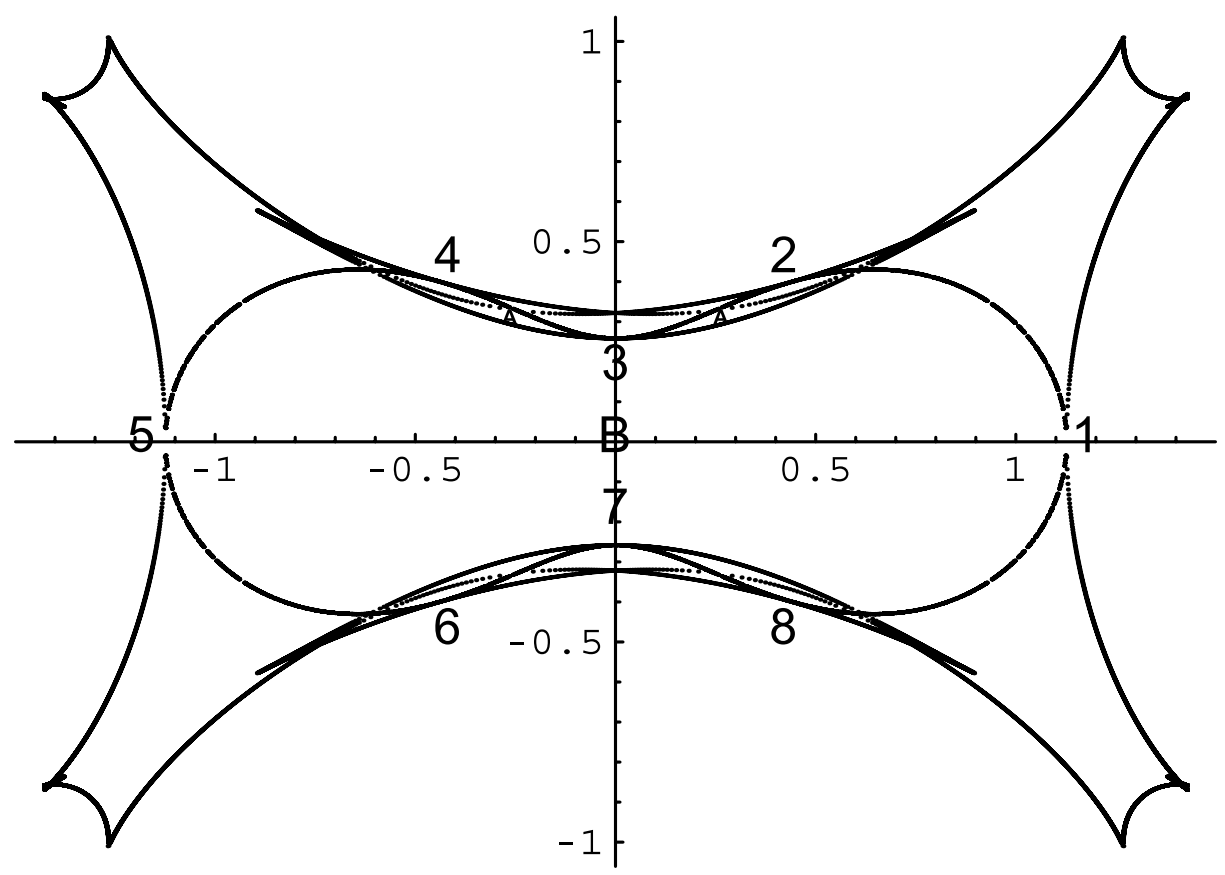

Figure 10. Preimage of $f(S)$ for $f(z)=-\frac{1}{2} z^{3}+\frac{9}{10} z-\bar{z}$ 
To make further progress with this, we need to have a better understanding of what the regions of the partitions look like (for example, is a component of the partition of the image ever an annulus?) An obvious starting point is to look at harmonic polynomials where $C(f, \infty)=\varnothing$. We make the following

Conjecture. Let $f(z)=p(z)-\bar{z}$ with $p(z)$ being a polynomial in $z$ of degree two or higher. Let $\left\{R_{j}\right\}$ be a finite collection of bounded regions in the unbounded component of $\mathbb{C} \backslash S$ such that each $R_{j}$ is a component of $\mathbb{C} \backslash f^{-1}(f(S))$. Let $R=$ $\operatorname{int}\left(\bigcup \overline{R_{j}}\right)$. Suppose that we choose the $R_{j}$ such that $R$ is a connected region where

$z \in \partial R$ implies one of the following holds: (i) $z \in S$, or (ii) every neighborhood of $z$ contains points mapped to the unbounded component of $\mathbb{C} \backslash f(S)$. Then $f$ is univalent on $R$. Moreover, $R$ is maximal in the sense that if we add another bounded component of $\mathbb{C} \backslash f^{-1}(f(S))$ to $R$, then $f$ will not be univalent on the combined region.

\section{Global valence And LyzzaiK's local Results}

The examples with $\operatorname{Val}(f)<\infty$ where $f$ is defined in $\mathbb{C}$ in Sections 4 and 5 above share a common feature: the valence increases by an even number when one crosses an arc of $f(S)$ into the region containing the tangent line to that arc. Since $\operatorname{Val}(f)<\infty$ in each such example, these are examples of light harmonic functions. We shall apply some results of Lyzzaik Lyz92 concerning the behavior of a light harmonic function defined in a simply connected domain to show that the change in valence illustrated in these examples is a property of light harmonic functions in $\mathbb{C}$.

First, we will consider the setting for Lyzzaik's results. Let $W$ be a simply connected domain in $\mathbb{C}$ and $f$ a harmonic function in $W$. Then we can represent $f$ as $f=h+\bar{g}$ where $h$ and $g$ are holomorphic in $W$. Let $\psi(z)=\left(h^{\prime} / g^{\prime}\right)(z)$ for $z \in W ; \psi$ is the reciprocal of the dilatation of $f$. Lyzzaik denotes the critical set of $f$ by $J$; for consistency, we will denote it by $S$.

Definition 6.1 (Lyzzaik). For a light harmonic mapping $f$ in $W$ every $z \in N=$ $\{z \in S:|\psi(z)| \neq 1\}$ is called a non-folding critical point of $f$. Note that if $z \in N$, then $h^{\prime}(z)=g^{\prime}(z)=0$.

Lemma 6.2 (Lyzzaik). Every $z_{0} \in N$ belongs to a neighborhood that contains no other point in $S . \psi$ is unimodular on $\Gamma_{f}=S \backslash N$ and $\Gamma_{f}$ consists of curves which are analytic except possibly for algebraic singularities.

Lyzzaik parametrizes a directed Jordan subarc $\gamma$ of $\Gamma_{f}$ by an analytic path $z(t)$ for $t \in I=[0,1]$. He then defines a continuous, increasing function $\phi: I \rightarrow \mathbf{R}$ by requiring that $\psi(z(t))=\exp (i \phi(t))$. An easy calculation shows that

$$
\frac{d}{d t} f(z(t))=2(\operatorname{Re} \omega(t)) \exp (i \phi(t) / 2)
$$

where

$$
\omega(t)=h^{\prime}(z(t)) z^{\prime}(t) \exp (-i \phi(t) / 2)
$$

for $z \in \gamma \subseteq S \backslash N$. 
Definition 6.3 (Lyzzaik). Let $f$ be a light harmonic mapping in $W$ and let $z_{0} \in \Gamma_{f}$. (A) If $\psi^{\prime}\left(z_{0}\right) \neq 0$, then $z_{0}$ is interior to a Jordan subarc $\gamma$ of $\Gamma_{f}$ which we assume given as above, with $z_{0}=z\left(t_{0}\right), 0<t_{0}<1$. Let $\omega(t)$ be as above. We call $z_{0}$ a critical point of $f$ of the

(a) first kind if $R e \omega$ changes sign at $t_{0}$;

(b) second kind if $z_{0}$ is not a critical point of the first kind and if $z_{0}$ is a zero of $h^{\prime}$, or equivalently $g^{\prime}$ (which yields $\left.R e \omega\left(t_{0}\right)\right)=0$ ).

(B) If $\psi^{\prime}\left(z_{0}\right)=0$, then we call $z_{0}$ a critical point of $f$ of the third kind.

Let $F_{j}$ where $j=1,2$, or 3 , denote the set of all critical points of $f$ of the $j$ th kind, and let $F=\bigcup_{j=1}^{3} F_{j}$. We call $F$ the set of folding critical points of $f$. Note that this classification is independent of $\gamma$ and its parametrization.

Theorem 6.4 (Lyzzaik). Let $f$ be a light harmonic function in $W$. Then $F \cup N$ consists of isolated points.

Remark 6.5. We also claim that $F \cup N$ has no point of accumulation in $W$. Since $f$ is light, neither $F_{3}$ nor $N$ can have a point of a accumulation; otherwise $S$ has non-empty interior (in which case, the proof of Lemma 4.7 implies that $f$ is not light). Further, as noted by Lyzzaik, Re $\omega$ can have only finitely many zeros on any Jordan arc in $S$ when $f$ is light. Hence, $F_{1} \cup F_{2}$ has no point of accumulation.

We can now state one of Lyzzaik's structure theorems for the local behavior of $f$ near the critical set. The notation $f_{z_{0}} \sim z^{j}, \bar{z}^{k}$ is explained in Section 2.1 above.

Theorem 6.6 (Lyzzaik). Let $f=\bar{g}+h$ be a light harmonic mapping in $W, z_{0} \in \Gamma_{f}$, and $\ell \geq 0$ the order of $z_{0}$ as a zero of $h^{\prime}$ or equivalently $g^{\prime}$.

(a) Suppose that $z_{0} \in \Gamma_{f} \backslash\left(F_{1} \cup F_{3}\right)$. Then $f$ satisfies $f_{z_{0}} \sim z^{\ell+1}, \bar{z}^{\ell+1}$ if $\ell$ is even (including zero), and $f_{z_{0}} \sim z^{\ell+2}, \bar{z}^{\ell}$ or $f_{z_{0}} \sim z^{\ell}, \bar{z}^{\ell+2}$ if $\ell$ is odd.

(b) Suppose that $z_{0} \in F_{1}$. Then $f$ satisfies $f_{z_{0}} \sim z^{\ell+1}, \bar{z}^{\ell+3}$ or $f_{z_{0}} \sim$ $z^{\ell+3}, \bar{z}^{\ell+1}$ if $\ell$ is even, and $f_{z_{0}} \sim z^{\ell+2}, \bar{z}^{\ell+2}$ if $\ell$ is odd.

Lyzzaik defines a convex arc to be a directed simple arc that has the slope of its tangent continuously increasing. An arc is locally convex at $z_{0}$ if $z_{0}$ belongs to some open subarc which is convex. Let $\gamma \subseteq \Gamma_{f}$ and $z_{0} \in$ int $\gamma$. Lyzzaik examines arg $\frac{d}{d t} f(z(t))$ at $z_{0}=z\left(t_{0}\right)$ and shows that $f(\gamma)$ is locally convex at $z_{0} \in \Gamma_{f} \backslash\left(F_{1} \cup F_{3}\right)$. Thus we can speak of the region which contains the tangent line to $f(\gamma)$ at $f\left(z_{0}\right)$ if $z_{0} \notin F$. (Another approach is to note that if $\gamma \subseteq \Gamma_{f}$, then $f(\gamma)$ cannot be piecewise constant; otherwise, a connected subset of $\gamma$ is mapped to a point and $f$ is not light. One can then apply a result of P. Duren and D. Khavinson DK97] to show that $f(\gamma)$ is concave, provided that $f$ is univalent in a region with $\gamma$ in its boundary. However, this approach requires us to show that we can find such a region of univalence.)

Let $f$ be a light harmonic function in $\mathbb{C}$. Let $\gamma$ be a simple analytic arc in the critical set such that $\beta=f(\gamma)$ is convex (and contains no points of inflection) and such that $\gamma \cap(F \cup N)=\varnothing$. An arc will be called non-degenerate if it is a non-empty arc that does not consist of a single point. Here, $\gamma$ is non-degenerate. Then, given $z_{0} \in$ int $\gamma$, there is a neighborhood $U$ of $z_{0}$ such that $\gamma$ partitions $U$ into a sense-preserving region $U^{+}$and a sense-reversing region $U^{-}$. If we choose $z_{0} \in$ int $\gamma$, then $\ell=0$ in Theorem [6.6 (since $g^{\prime}\left(z_{0}\right) \neq 0$ ) and $f_{z_{0}} \sim z, \bar{z}$. By Theorem 6.6 and its proof, we can choose $U$ and some neighborhood of $f\left(z_{0}\right)$, say 
$B$, such that $\beta$ partitions $B$ into two regions $V^{+}$and $V^{-}$and such that $f$ maps $U^{+}$ 1-1 onto $V^{+}$and maps $U^{-} 1-1$ onto $V^{+}$. Here, $V^{+}$is chosen such that the tangent to $\beta$ at $f\left(z_{0}\right)$ lies in $V^{+}$. We will use these ideas to prove:

Theorem 6.7. Let $f$ be a finite valence harmonic mapping on $\mathbb{C}$. Let $\Omega_{+}$and $\Omega_{-}$ be distinct components of $\mathbb{C} \backslash(f(S) \cup C(f, \infty))$ that share a common non-degenerate boundary arc $\beta_{0}$. Suppose that int $\beta_{0}$ does not lie completely in $C(f, \infty)$. Then there is a non-degenerate subarc $\beta$ of $\beta_{0}$ that lies outside of $C(f, \infty)$ and such that $f^{-1}(\beta) \cap(F \cup N)=\varnothing$. Choose $w_{0} \in$ int $\beta$ and choose $\Omega_{+}$to be the region containing the tangent to $\beta$ at $w_{0}$. Suppose that $w_{0}$ has $N_{0} \geq 0$ distinct preimages off $S$ and $N_{1}>0$ distinct preimages in $S$. Then $\operatorname{Val}\left(f, \Omega_{-}\right)=N_{0}$ and $\operatorname{Val}\left(f, \Omega_{+}\right)=$ $N_{0}+2 N_{1}$.

Proof. Since $f$ has finite valence, $f$ is light. We wish to choose a non-degenerate, simple analytic arc $\beta \subseteq \beta_{0}$ such that $\beta \cap C(f, \infty)=\varnothing$, with $\beta$ in the boundary between two regions of constant valence, and such that $\beta \cap f(F \cup N)=\varnothing$. The two regions are non-empty by Corollary 4.10, since $\operatorname{Val}(f)<\infty$. By our choice of $\beta$, a structure theorem of Lyzzaik for light harmonic functions (Theorem 6.6) should help us count the distinct preimages of points in some sufficiently small neighborhood $B$ of $w_{0}$.

(1) Existence of $\beta$ : Since int $\beta_{0}$ does not lie completely in $C(f, \infty)$, we can find a bounded, closed, non-degenerate subarc $\tilde{\beta}$ such that $\exists w \in$ int $\tilde{\beta}$ with $w \notin C(f, \infty)$. Since $C(f, \infty)$ is closed, there exists a neighborhood of $w$ that is disjoint from $C(f, \infty)$. Take $\beta_{1}$ to be a closed, non-degenerate subarc of $\tilde{\beta}$ contained in this neighborhood and to have $w$ in its interior.

If $K$ is an arbitrary compact set, then $(F \cup N) \cap K$ must be finite by Remark 6.5. Since $\beta_{1} \cap C(f, \infty)=\varnothing$, the closed set $f^{-1}\left(\beta_{1}\right)$ is also bounded. Thus, $f^{-1}\left(\beta_{1}\right)$ is compact and $\beta_{1}$ contains at most a finite number of points with preimages in $F \cup N$. So, we may find a bounded subarc $\beta$ of $\beta_{0}$ that neither intersects $C(f, \infty)$ nor contains points in $f(F \cup N)$. Moreover, we may assume that this arc is convex and without any points of inflection. Since $f$ has finite valence, $f(S) \cup C(f, \infty)$ has empty interior. By construction, $\beta$ will be the local boundary between $\Omega_{+}$and $\Omega_{-}$.

(2) Preimages of $w_{0}$ : Choose $w_{0} \in$ int $\beta$. Let $f^{-1}\left(w_{0}\right) \cap S=\left\{z_{1}, z_{2}, \ldots, z_{N_{1}}\right\}$. In a sufficiently small neighborhood of $z_{j}, S$ consists of a single non-degenerate arc since $z_{j} \notin N \cup F_{3}$ by (1). A non-degenerate subarc of this arc is mapped to a subarc of $\beta$. This follows from our assumption that $\beta_{0}$ locally separates components of the partition; i.e., if two distinct curves in $f(S)$ cross at $w_{0}$, then $\beta_{0}$ doesn't separate $\Omega_{+}$and $\Omega_{-}$in a sufficiently small neighborhood of $w_{0}$. Since $w_{0} \in f(S)$, we have $N_{1}>0$. Let $f^{-1}\left(w_{0}\right) \cap$ $(\mathbb{C} \backslash S)=\left\{\zeta_{1}, \zeta_{2}, \ldots, \zeta_{N_{0}}\right\}$, where $N_{0} \geq 0$.

(3) Construction of neighborhood $B$ of $w_{0}$ : Consider a preimage $\zeta_{j}$ of $w_{0}$ off of $S$. By the inverse function theorem, there exist $Q_{j}$, an open neighborhood of $\zeta_{j}$, and $V_{j}$, an open neighborhood of $w_{0}$, such that $f: Q_{j} \rightarrow V_{j}$ is 1-1, onto. Without loss of generality, we may assume $Q_{j} \subseteq \mathbb{C} \backslash f^{-1}(f(S) \cup C(f, \infty))$. Now consider a preimage $z_{j}$ in $S$. By construction, $z_{j} \notin N \cup F_{3}$. Let $\gamma$ be a simple arc in $S$ such that $z_{j} \in$ int $\gamma$ and $f(\gamma) \subseteq \beta$. By our choice of $\beta, \gamma \cap(F \cup N)=\varnothing$. Hence, $g^{\prime}\left(z_{j}\right) \neq 0$. We may apply our interpretation 
above of Theorem 6.6 to find an open neighborhood $U_{j}$ of $z_{j}$ and an open neighborhood $W_{j}$ of $w_{0}$ such that:

- $\gamma$ partitions $U_{j}$ into a sense-preserving region $U_{j}{ }^{+}$and a sense-reversing region $U_{j}^{-}$.

- $U_{j}{ }^{+}$and $U_{j}{ }^{-}$are each mapped 1-1 onto $W_{j} \cap \Omega_{+}$.

Let $B_{0}=\bigcap_{j=1}^{N_{0}} V_{j}$ and let $B_{1}=\bigcap_{j=1}^{N_{1}} W_{j}$. Clearly, $B_{1} \neq \varnothing$. If $N_{0}>0$, $B_{0} \neq \varnothing$, so let $B=B_{0} \cap B_{1}$. Otherwise, $B_{0}$ is empty, so let $B=B_{1}$. By construction, $B$ is open. Finally, let $T=f^{-1}(B) \cap\left(\left(\bigcup_{j=1}^{N_{0}} Q_{j}\right) \cup\left(\bigcup_{j=1}^{N_{1}} U_{j}\right)\right)$. Then every preimage of $w_{0}$ is in int $T$. We may choose the $Q_{j}$ and the $U_{j}$ such that these sets are all pairwise disjoint.

(4) Valence in $\Omega_{+}$: By construction, each $w \in B \cap \Omega_{+}$has exactly $N_{0}+2 N_{1}$ distinct preimages in $T$ and thus has at least $N_{0}+2 N_{1}$ distinct preimages in $\mathbb{C}$. We claim that $\exists w_{+} \in B \cap \Omega_{+}$such that $\operatorname{Val}\left(f, w_{+}\right)=N_{0}+2 N_{1}$. Suppose not. Then $\exists\left\{w_{n}\right\} \subset B \cap \Omega_{+}$such that $w_{n} \rightarrow w_{0}$ and such that each $w_{n}$ has at least $N_{0}+2 N_{1}+1$ distinct preimages in $\mathbb{C}$. In particular, each $w_{n}$ has a preimage $\xi_{n} \notin T$. Since $w_{n} \rightarrow w_{0}$ and $w_{0} \notin C(f, \infty),\left\{\xi_{n}\right\}$ is bounded, so has a convergent subsequence, which we will also denote $\left\{\xi_{n}\right\}$. Let $\xi_{n} \rightarrow \xi_{0}$. Since $f\left(\xi_{0}\right)=w_{0}, \xi_{0} \in$ int $T$. Thus, $\xi_{n} \in T$ for $n$ sufficiently large, a contradiction. Thus, $\exists w_{+} \in B \cap \Omega_{+}$such that $\operatorname{Val}\left(f, w_{+}\right)=N_{0}+2 N_{1}$. Since, by Theorem 4.1, the valence of $f$ is constant on the region $\Omega_{+}, \operatorname{Val}\left(f, \Omega_{+}\right)=\operatorname{Val}\left(f, w_{+}\right)=N_{0}+2 N_{1}$.

(5) Valence in $\Omega_{-}$: By construction, each $w \in B \cap \Omega_{-}$has exactly $N_{0}$ distinct preimages in $T$ (one preimage in each $Q_{j}$ ) and thus has at least $N_{0}$ distinct preimages in $\mathbb{C}$. We claim that $\exists w_{-} \in B \cap \Omega_{-}$such that $\operatorname{Val}\left(f, w_{-}\right)=N_{0}$. Suppose not. Then $\exists\left\{w_{n}\right\} \subset B \cap \Omega_{-}$such that $w_{n} \rightarrow w_{0}$ and such that each $w_{n}$ has at least $N_{0}+1$ preimages in $\mathbb{C}$. In particular, each $w_{n}$ has a preimage $\xi_{n} \notin f^{-1}(B) \cap\left(\bigcup_{j=1}^{N_{0}} Q_{j}\right)$. Since $w_{0} \notin C(f, \infty),\left\{\xi_{n}\right\}$ is bounded, so has a convergent subsequence, which we will also denote $\left\{\xi_{n}\right\}$. Let $\xi_{n} \rightarrow \xi_{0}$. Since $f\left(\xi_{0}\right)=w_{0}, \xi_{0} \in$ int $T$. Thus, for $n$ sufficiently large, $\xi_{n} \in T$. Since $f$ maps $\bigcup_{j=1}^{N_{1}} U_{j}$ into $\beta \cup \Omega_{+}, \xi_{n} \in f^{-1}(B) \cap\left(\bigcup_{j=1}^{N_{0}} Q_{j}\right)$ for sufficiently large $n$, a contradiction. Thus, $\exists w_{-} \in B \cap \Omega_{-}$such that $\operatorname{Val}\left(f, w_{-}\right)=N_{0}$. Since the valence of $f$ is constant on the region $\Omega_{-}$(Theorem 4.1 again), $\operatorname{Val}\left(f, \Omega_{-}\right)=\operatorname{Val}\left(f, w_{-}\right)=N_{0}$.

Remark 6.8. The proof above can also be extended to the case where $f$ is a finitevalence, light harmonic function in a simply connected, open set. In that case, $C(f)$ replaces $C(f, \infty)$ and $S$ is defined only for points in our open set.

Example 6.9. Consider Example 4.14 above. We can select a few points in each region of the partition and ask Mathematica to find the preimages in $B(0,1)$. $\operatorname{Val}(f, w)=0$ in the unbounded component of $\mathbb{C} \backslash f(S)$. In each "point of the star" region, $\operatorname{Val}(f, w)=1 . \operatorname{Val}(f, w)=2$ in the "center of the star" region (recall that the origin is in $f(S)$ and is not in this region). The origin has one preimage in $B(0,1)$. This is not a counterexample to this extended version of Theorem 6.7 because the "star" is $C(f)$, not $f(S)$. Effectively, when we restrict $f$ to our region, there are no points with $|z|>1$ for $f$ to "fold" over $|z|=1$. 


\section{ACKNOWLEDGEMENTS}

Many of the results in this paper appeared in the author's thesis [Neu03]. The author is grateful to her advisor, D. Sarason, for encouraging her to study planar harmonic functions. She also wishes to thank him for his open-mindedness, patience, and kindness in helping her learn about mathematics and research. His many suggestions were essential to this work. The author is indebted to W. Hengartner for suggesting that her earlier results concerning regions of constant valence should be extendable to the case of infinite valence, and to H. Helson for suggesting that they should be extendable to the case of functions in arbitrary open regions (not just in the entire plane.) The author is also indebted to D. Khavinson for information concerning the Brelot-Choquet Lemma. The author also wishes to thank A. Bakke for adding labels to some of the EPS files generated by Mathematica.

\section{REFERENCES}

[AL88] Y. Abu-Muhanna and A. Lyzzaik, A geometric criterion for decomposition and multivalence, Math. Proc. Cambridge Phil. Soc. 103 (1988), 487-495. MR 89e:30010

[Bal91] Mark Benevich Balk, Polyanalytic Functions, Mathematical research, volume 63, Akademie Verlag GmbH (1991). MR 93k:30076

[BC55] Marcel Brelot and Gustave Choquet, Polynômes harmoniques et polyharmoniques, Second colloque sur les équations aux dérivées partielles, Bruxelles, 1954, pp. 45-66. Georges Thone, Liège; Masson \& Cie (1955). MR 16:1108e

[BHN99] Daoud Bshouty, Walter Hengartner, and M. Naghibi-Beidokhti, p-valent harmonic mappings with finite Blaschke dilatations, XII-th Conference on Analytic Functions (Lublin, 1998), Ann. Univ. Mariae Curie-Sklodowska Sect. A, 53 (1999), 9-26. MR 2001j:30016

[BHS95] Daoud Bshouty, Walter Hengartner, and Tiferet Suez, The exact bound on the number of zeros of harmonic polynomials, J. Anal. Math. 67 (1995), 207-218. MR 97f:30025

[CL66] E. F. Collingwood and A. J. Lohwater, The Theory of Cluster Sets, Cambridge University Press (1966). MR 38:325

[DK97] Peter Duren and Dmitry Khavinson, Boundary correspondence and dilatation of harmonic mappings, Complex Variables Theory Appl. 33 (1997), 105-111. MR 98m:30039

[KS03] Dmitry Khavinson and Grzegorz Świạtek, On the number of zeros of certain harmonic polynomials, Proc. Amer. Math. Soc. 131 (2003), 409-414.

[Lew36] Hans Lewy, On the non-vanishing of the Jacobian in certain one-to-one mappings, Bull. Amer. Math. Soc. 42 (1936), 689-692.

[Lyz92] Abdallah Lyzzaik, Local properties of light harmonic mappings, Canad. J. Math. 44 (1992), 135-153. MR 93e:30048

[Mun75] James R. Munkres, Topology: A First Course, Prentice-Hall, Inc. (1975). MR 57:4063

[Neu03] Genevra Chasanov Neumann, Valence of harmonic functions, Ph.D. dissertation, University of California, Berkeley. 2003.

[OS86] M. Ortel and W. Smith, A covering theorem for continuous locally univalent maps of the plane, Bull. London Math. Soc. 18 (1986), 359-363. MR 88b:30013

[Smi71] Kennan T. Smith, Primer of Modern Analysis, Bogden \& Quigley, Inc. (1971). MR 84m:26002

[Sto56] S. Stöilow, Leçons sur les principes topologiques de la theórie des fonctions analytiques, deuxième edition, Gauthier-Villars (1956). MR 18:568b

[ST00] T. J. Suffridge and J. W. Thompson, Local behavior of harmonic mappings, Complex Variables Theory Appl. 41 (2000), 63-80. MR 2001a:30019

[Wil94] Alan Stephen Wilmshurst, Complex harmonic mappings and the valence of harmonic polynomials, D.Phil. thesis, University of York, England. 1994.

[Wil98] A. S. Wilmshurst, The valence of harmonic polynomials, Proc. Amer. Math. Soc. 126 (1998), 2077-2081. MR 98h:30029

Department of Mathematics, University of California, Berkeley, California 94720

Current address: Department of Mathematics, Kansas State University, Manhattan, Kansas 66506

E-mail address: neumann@math.ksu.edu 